\title{
Transmission loss of plates with multiple embedded acoustic black holes using statistical modal energy distribution analysis
}

\author{
Jie Deng ${ }^{\mathrm{a}, \mathrm{b}}$, Oriol Guasch ${ }^{\mathrm{b}, *}$, Laurent Maxit ${ }^{\mathrm{c}}$, Ling Zheng ${ }^{\mathrm{a}}$ \\ ${ }^{a}$ State Key Laboratory of Mechanical Transmission, College of Automotive Engineering \\ Chongqing University, Chongqing 400044, PR China \\ ${ }^{b}$ GTM - Grup de Recerca en Tecnologies Mèdia, La Salle, Universitat Ramon Llull \\ C/ Quatre Camins 30, 08022 Barcelona, Catalonia (Spain) \\ ${ }^{c}$ INSA-Lyon, Laboratoire Vibrations-Acoustique (LVA) \\ 25 bis, av. Jean Capelle, F-69621 Villeurbanne Cedex, France
}

\begin{abstract}
Acoustic black holes (ABHs) in beams and plates have been extensively studied as a passive method for vibration attenuation and noise reduction. However, most research to date has focused on analyzing the behavior of a single ABH structural element, using numerical or semi-analytical deterministic approaches. If $\mathrm{ABHs}$ are to be exploited for practical industrial applications, there is a need to characterize their performance in complex built-up structures and to describe them with statistical methods in the mid-high frequency range. This paper presents a first step towards this goal by employing statistical modal energy distribution analysis (SmEdA) to evaluate the transmission loss of ABH panels separating two air cavities. SmEdA splits vibroacoustic systems into subsystems and establishes power balance equations between the modes belonging to different subsystems. This avoids the energy equipartition assumption of traditional statistical energy analysis (SEA) and extends it to low modal overlap systems. In this work, the benefits of embedding ABHs on plates for noise reduction between cavities are predicted with SmEdA. The role played by the size, shape and number of $\mathrm{ABH}$ indentations on the plate are inspected, as well as the influence of other parameters like the truncation thickness, ABH order and damping. The effects of added mass and stiffness of the damping layer are investigated and it is observed that, with proper design, ABH plates can exhibit substantial transmission loss improvement in the vicinity of the critical frequency of uniform plates. Both resonant and non-resonant transmission are considered in the analysis.
\end{abstract}

Keywords: Acoustic black holes (ABHs), Mid-high frequency modelling, Transmission Loss, Statistical modal energy distribution analysis (SmEdA), Gaussian expansion method (GEM)

\section{Introduction}

Acoustic black holes (ABHs) have revealed as an effective way to suppress structural vibrations and noise in mechanical systems. The ABH effect relies on removing material from beams and plates by tailoring indentations that follow a power-law thickness variation [1-3], or by altering the inner ring radii of thinwall separated cavities in duct terminations [4-7]. ABH profiles have the special feature of slowing down the propagation speed of input waves, which concentrate their energy at the center (plates) or termination (beams and ducts) of the ABH element. There, energy can be dissipated by means of different configurations of viscoleastic damping layers [8-10]. The ABH effect has proved to be a very efficient method to reduce flexural vibrations (see e.g., [11-16]) and noise radiation [17-20] in beams and plates. Furthermore, arrays of $\mathrm{ABH}$ s have shown amazing properties for wave manipulation in plates [21-23]. Manufacturing considerations can be found e.g., in [24, 25].

*Corresponding Author: oriol.guasch@salle.url.edu 
To date, the $\mathrm{ABH}$ effect has been mostly analyzed for single structural elements and using deterministic approaches. The latter include numerical simulations, as those in the finite element method (FEM) which is sometimes combined with the boundary element method (BEM) to predict noise radiation [17]. Another option is semi-analytical modeling like in the Rayleygh-Ritz method with trigonometric [26], wavelet [11, 13] or Gaussian basis functions [10, 27]. Implementing ABHs in realistic environments demands, however, one step forward and start analyzing the functioning of ABH beams and plates as parts of more complex built-up structures. This also means that deterministic methods shall be abandoned in favor of statistical ones, like e.g., statistical energy analysis (SEA) [28-30], to perform simulations in the mid-high frequency range.

This paper proposes a first attempt in that direction by studying the energy noise reduction between two air cavities separated by a plate with embedded ABHs, using the statistical modal energy distribution analysis (SmEdA) developed in [31, 32]. Few works exist in literature dealing with compound systems that involve $\mathrm{ABH}$ structures. In [33, 34], for example, the case of an ABH plate coupled to a cavity was experimentally tested and compared with FEM simulations. Very recently, results on the transmission loss of rectangular and circular plates with $\mathrm{ABH}$ indentations were respectively reported in [35] and [36]. Again, measurements were compared with FEM/BEM models showing the benefits of embedding ABHs on the plate between cavities. Nonetheless, it is well-known that the computational cost of FEM/BEM models for complex systems becomes prohibitive as soon as frequencies become large and that the system dynamics gets very sensitive to variations in the structural parameters. Statistical methods can circumvent such problems. As said, SmEdA has been chosen to analyze the transmission loss of an ABH plate in this work. SmEdA was designed to address the so-called mid-frequency problem, at which deterministic FEM/BEM is very expensive and the high frequency assumptions of SEA are too restrictive. Several other methods have been proposed in literature to cover the mid frequency gap by relaxing SEA hypotheses: from energy distribution models [37, 38] to the asymptotic scaled modal analysis (ASMA) [39, 40], among many others (see, e.g., [41, 42]). As in SEA, SmEdA splits a mechanical system into connected subsystems that satisfy specific modal coupling assumptions (see e.g., [43, 44]). However, the power balance equations are not established between subsystems in SmEdA, but among modes belonging to different subsystems. This allows one to apply SmEdA to the cases of low modal overlap, locally excited subsystems and to consider complex heterogeneous subsystems with dissipative materials as well [43, 45].

The determination of subsystem modes constitutes the first step of SmEdA. Therefore, one may wonder which boundary conditions should be considered to find them. In the case at hand, which involves a flexible structure (plate) coupled to acoustic closed cavities, the dual modal formulation (DMF) [31, 46, 47] offers a clear answer to the question. To fulfill the modal coupling assumptions, the plate shall be characterized by the displacement field and uncoupled-free modes, while the cavities must be described by the acoustic pressure field and blocked modes (i.e. homogeneous Neumann boundary conditions; cavity modes are assumed to have zero acoustic particle velocity at all walls, including the separating plate). It is to be mentioned that such division is not so straightforward when there is no clear impedance mismatch between subsystems, as it may occur at the junctions of multiple connected subsystems [44, 48]. In the current example, rectangular acoustic cavities will be considered so the blocked modes at the frequency bands of interest can be computed from standard analytical formulas. In what concerns the uncoupled-free modes of the plate with embedded ABHs, we will resort to the Gaussian expansion method (GEM), which has proven very fast and precise in previous works on ABHs [10, 27, 49]. The GEM relies on expanding the plate displacement field in terms of Gaussian shape functions, in the framework of the Rayleigh-Ritz method. Once the cavity and plate modes are known, power balance equations can be established among them yielding the final form of a SmEdA matrix system. The latter has much smaller dimensions than a FEM/BEM system and can be solved and exploited for parametric analyzes in the mid-high frequency range. In the presented examples, it will be checked how the size, shape and number of ABHs influence noise reduction between cavities. Further issues, such as the effects of $\mathrm{ABH}$ parameters like the truncation thickness, $\mathrm{ABH}$ order and damping will be also investigated. Both resonant and non-resonant transmission will be taken into account in the simulations $[50,51]$.

The remaining of the manuscript is organized as follows. In Section 2, we state the transmission loss problem to be solved. The theoretical formulation is presented in Section 3, where it is first shown how to compute the $\mathrm{ABH}$ free modes using the GEM and the cavity blocked modes from analytical formulas. 
(a) Source cavity $14^{y} \quad$ Receiver cavity 2
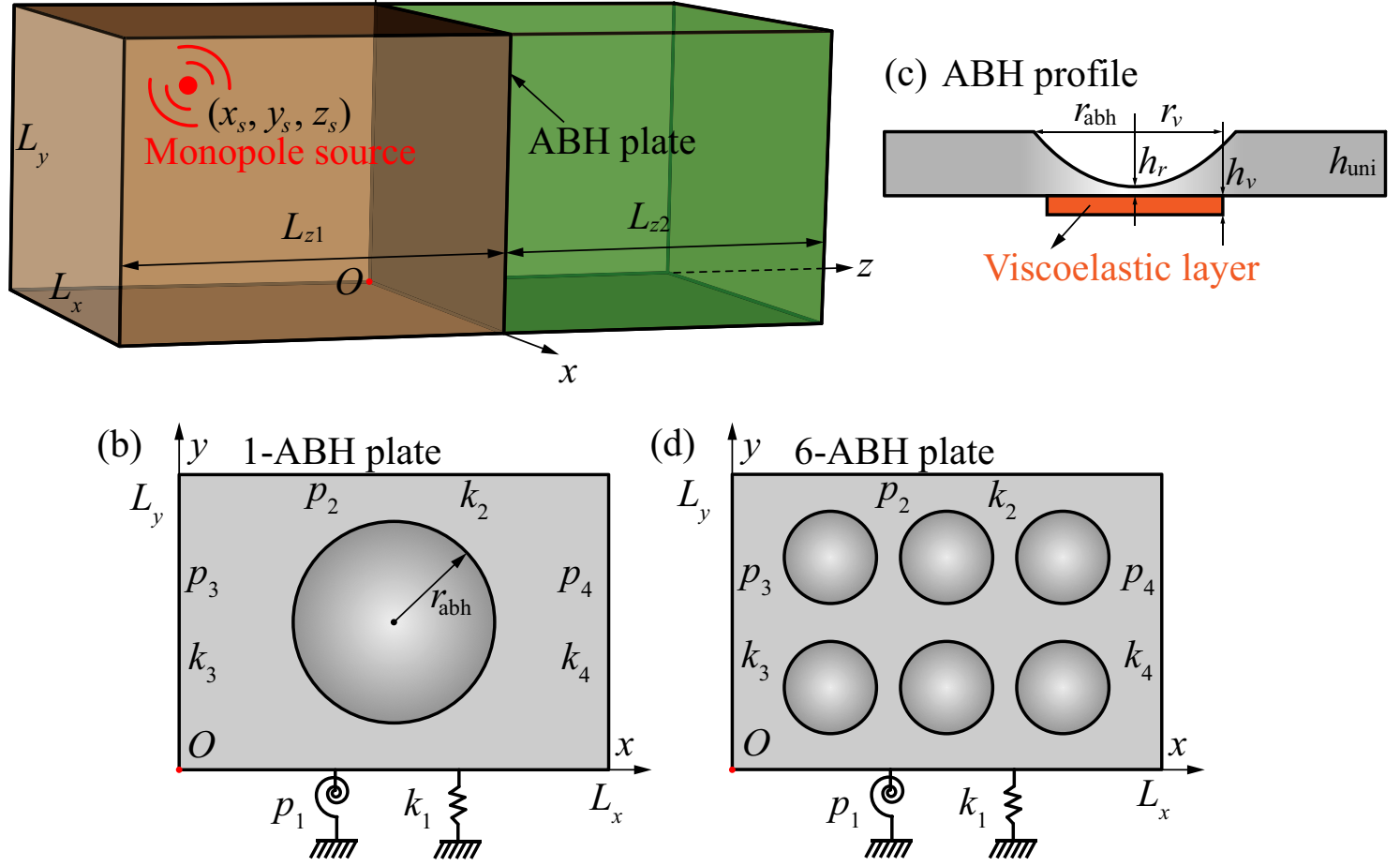

(d) $\uparrow y \quad 6-\mathrm{ABH}$ plate

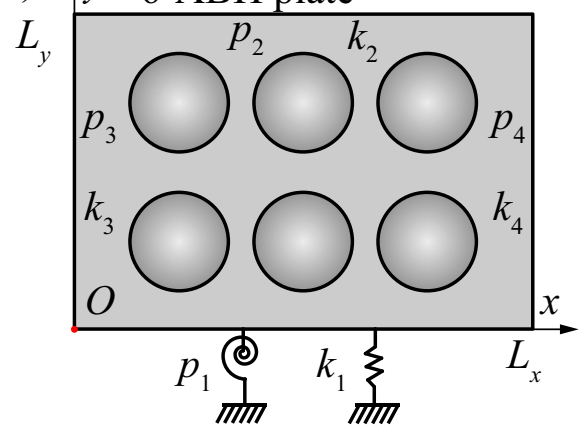

Figure 1: Illustration of (a) the cavity-plate-cavity system, (b) 1-ABH plate with translational and rotational stiffness boundary conditions at each side, (c) cross-section of an ABH plate profile including the damping layer, (d) a 6-ABH plate.

Then, to facilitate comprehension, a constructive approach is followed to expose SmEdA power balance equations and explain how to compute subsystem modal energies. These can be used to obtain the energy noise reduction between cavities and therefore the $\mathrm{ABH}$ plate transmission loss. Section 4 contains the results from numerical simulations. The noise reduction for a separating plate with single and multiple $\mathrm{ABH}$ indentations is calculated and compared to that of uniform plates. A parametric analysis follows and explanations are given on the benefits and reasons why ABHs help improving the transmission loss of plates. Conclusions close the paper in Section 5.

\section{Statement of the problem: cavity-ABH plate-cavity system}

Let us consider two rectangular cavities, the source cavity (Cavity 1) with depth $L_{z 1}$ and the receiver cavity (Cavity 2) with depth $L_{z 2}$, separated by a wall of surface $L_{x} \times L_{y}$ and thickness $h_{\text {uni }}$ (see Fig. 1a). As illustrated in the figure, a monopole sound source is located in the source cavity at position $\boldsymbol{x}_{s}=\left(x_{s}, y_{s}, z_{s}\right)^{\top}$. Except for the partition plate, all other walls in cavities 1 and 2 are assumed perfectly rigid to prevent flanking path transmission. To enhance the partition plate transmission loss, we contemplate tailoring a circular ABH indentation on it (see Fig. 1b). The plate thickness in the ABH area decreases according to the power law $h(x, y)=\varepsilon r_{\mathrm{abh}}^{m}+h_{r}$, where $\varepsilon=\left(h_{\mathrm{uni}}-h_{r}\right) r_{\mathrm{abh}}^{-m}$ is the so-called ABH smoothness parameter, $r_{\text {abh }}$ represents the $\mathrm{ABH}$ radius, $h_{r}$ symbolizes the residual thickness and $m$ denotes the $\mathrm{ABH}$ order (see the cross-section in Fig. 1c). When a bending wave packet enters the ABH region, its group velocity slows down and its amplitude grows as the $\mathrm{ABH}$ center approaches. The wave packet can be strongly dissipated by attaching a viscoelastic layer of radius $r_{v}<r_{\text {abh }}$ in the ABH central area (see Fig. 1c and e.g., [8-10]).

The SmEdA model for the current problem consists of three subsystems, namely Cavity 1 , the ABH plate and Cavity 2. As mentioned in the Introduction, in SmEdA blocked modes are to be considered for the cavities (all walls are assumed rigid to compute them) while free modes are needed for the partition 
plate (the acoustic load from the cavities is neglected). However, one may still consider assigning specific conditions to the plate boundaries (e.g., clamped or simply supported) to simulate the transmission loss between dwellings in a building, or between cavities in a lab acoustic chamber where the panel is supported by elastic junctions. To this purpose, one can make use of uniformly distributed artificial springs with different stiffness to emulate various boundary conditions [52]. As shown in Fig. 1b, the coefficient $p_{1}$ stands for the rotational stiffness at the plate bottom side, while $k_{1}$ refers to the translational stiffness at the same boundary. Analogous conditions and notation can be assigned to the remaining three plate sides, namely 2 , 3 , and 4 , though the springs are not explicitly plotted in the figure.

Next section's goal is to establish the SmEdA power balance equations for the cavity-ABH plate-cavity system to compute the noise reduction from Cavity 1 to Cavity 2. Separating walls embedding multiple $\mathrm{ABH}$ instead of one will be also discussed for comparison (a sketch for a $6 \mathrm{ABH}$ plate is given in Fig. 1d). The $1 / 3$ octave frequency bands from $400 \mathrm{~Hz}$ to $6300 \mathrm{~Hz}$ will be considered for the analysis.

\section{SmEdA model for the cavity-ABH plate-cavity system}

\subsection{Gaussian expansion method to extract the $A B H$ plate modes}

To compute the ABH plate modes use is made of the two-dimensional GEM approach in [27, 49]. The Kirchoff-Love theory for thin shells is assumed and only flexural displacements are taken into account. The displacement field, $w$, is expanded in terms of $2 \mathrm{D}$ Gaussian basis functions $\chi_{i}(x, y)$,

$$
w(x, y, t)=\sum_{i} a_{i}(t) \chi_{i}(x, y)=: \boldsymbol{a}^{\top} \chi=\hat{\boldsymbol{A}}^{\top} \chi \mathrm{e}^{\mathrm{i} \omega t},
$$

where in the last two equalities we have defined the vector of unknown weight coefficients, $\boldsymbol{a}=\hat{\boldsymbol{A}} \mathrm{e}^{\mathrm{i} \omega t}$, and that of basis functions, $\chi$. The vector $\chi$ can be separated into $x$ and $y$ components, namely $\chi=\boldsymbol{\alpha}_{x} \otimes \boldsymbol{\alpha}_{y}$, where $\otimes$ stands for the Kronecker product. The entries in $\boldsymbol{\alpha}_{x}$ and $\boldsymbol{\alpha}_{y}$ are Gaussian basis functions of the type,

$$
\alpha_{x}^{i}=2^{m_{x} / 2} \mathrm{e}^{-\left(2^{m_{x}} x-n_{x}^{i}\right)^{2} / 2}, \alpha_{y}^{i}=2^{m_{y} / 2} \mathrm{e}^{-\left(2^{m_{y}} y-n_{y}^{i}\right)^{2} / 2},
$$

in which $m_{x}$ and $m_{y}$ respectively represent scaling parameters to dilate the Gaussians in the $x$ and $y$ directions, while $n_{x}^{i}$ and $n_{y}^{i}$ are translating parameters to move the Gaussians along the coordinate axes.

Taking into account the finite factorization in Eq. (1), the kinetic, $K$, and potential, $U$, energies of the plate can be determined to build the Lagrangian,

$$
\mathscr{L}=K-U=-\frac{1}{2} \omega^{2} \hat{\boldsymbol{A}}^{\top} \boldsymbol{M} \hat{\boldsymbol{A}} \mathrm{e}^{\mathrm{i} 2 \omega t}-\frac{1}{2} \hat{\boldsymbol{A}}^{\top} \boldsymbol{K} \hat{\boldsymbol{A}} \mathrm{e}^{\mathrm{i} 2 \omega t} .
$$

For a plate of varying thickness, $h(x, y)$, the mass matrix $\boldsymbol{M}$ in Eq. (3) is given by

$$
\boldsymbol{M}=\int_{0}^{L_{y}} \int_{0}^{L_{x}} \rho h(x, y) \chi \chi^{\top} \mathrm{d} x \mathrm{~d} y,
$$

while the stiffness matrix $\boldsymbol{K}$ consists of two parts,

$$
\boldsymbol{K}=\boldsymbol{K}_{\text {plate }}+\boldsymbol{K}_{\text {edge }} .
$$

Here, $\boldsymbol{K}_{\text {plate }}$ designates the plate stiffness matrix

$$
\begin{array}{rl}
\boldsymbol{K}_{\text {plate }}=\int_{0}^{L_{y}} \int_{0}^{L_{x}} & D(x, y)\left[\partial_{x x}^{2} \chi \partial_{x x}^{2} \chi^{\top}+\partial_{y y}^{2} \chi \partial_{y y}^{2} \chi^{\top}\right. \\
& \left.+\nu\left(\partial_{x x}^{2} \chi \partial_{y y}^{2} \chi^{\top}+\partial_{y y}^{2} \chi \partial_{x x}^{2} \chi^{\top}\right)+2(1-\nu) \partial_{x y}^{2} \chi \partial_{y x}^{2} \chi^{\top}\right] \mathrm{d} x \mathrm{~d} y
\end{array}
$$



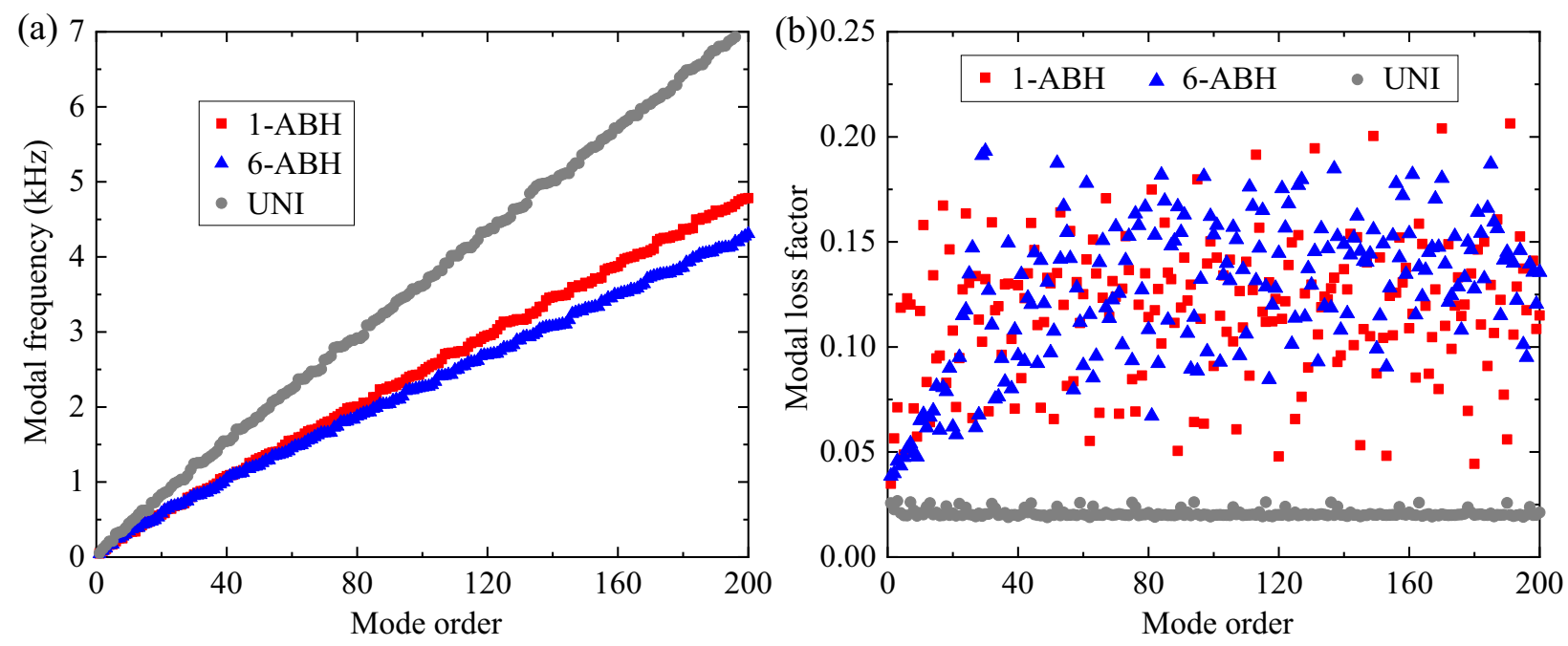

Figure 2: (a) Modal frequencies vs modal order for a 1-ABH plate, a 6-ABH plate and a uniform (UNI) plate. (b) Modal loss factors of the three plates.

with $D(x, y)=E(1+\mathrm{i} \eta) h^{3}(x, y) / 12\left(1-\nu^{2}\right)$ being the local flexural rigidity ( $E$ is the Young modulus and $\eta$ the plate material loss factor); $\nu$ stands for the Poisson ratio of the plate. The second matrix in Eq. (5) is the stiffness matrix that arises from boundary conditions, namely,

$$
\begin{aligned}
\boldsymbol{K}_{\text {edge }} & =\int_{0}^{L_{x}}\left[\left.\left(k_{1} \chi \chi^{\top}+p_{1} \partial_{x} \chi \partial_{x} \boldsymbol{\chi}^{\top}\right)\right|_{y=0}+\left.\left(k_{2} \chi \chi^{\top}+p_{2} \partial_{x} \chi \partial_{x} \boldsymbol{\chi}^{\top}\right)\right|_{y=L_{y}}\right] \mathrm{d} x \\
& +\int_{0}^{L_{y}}\left[\left.\left(k_{3} \chi \chi^{\top}+p_{3} \partial_{y} \chi \partial_{y} \chi^{\top}\right)\right|_{x=0}+\left.\left(k_{4} \chi \chi^{\top}+p_{4} \partial_{y} \chi \partial_{y} \chi^{\top}\right)\right|_{x=L_{x}}\right] \mathrm{d} y .
\end{aligned}
$$

The parameters $k_{i}(i=1 \ldots 4)$ represent the translational stiffness at each boundary (see Fig. 1), while $p_{i}$ $(i=1 \ldots 4)$ denote the rotational ones. As mentioned in section 2, assigning different values to $k_{i}$ and $p_{i}$ makes possible emulating various boundary conditions. Only the case of a simply supported panel will be addressed in this work for simplicity. That corresponds to taking values $k_{i}=+\infty$ and $p_{i}=0 \forall i$. Matrices $\boldsymbol{M}$ and $\boldsymbol{K}$ can be complemented to include viscoelastic layers attached to the plate, following the formulation in [27] (no to be detailed herein).

On the other hand, to compute the mass and stiffness matrices of ABH plates one can resort to the matrix replacing strategy, also proposed in [27]. First, analytical expressions for $\boldsymbol{M}$ and $\boldsymbol{K}$ are derived for a plate of uniform thickness $h_{\text {uni }}$. Then, the cylindrical portions of the plate corresponding to the regions where the ABHs are to be embedded are removed from $\boldsymbol{M}$ and $\boldsymbol{K}$ and substituted with the contributions of the ABHs and their corresponding viscoelastic layers. These contributions are computed numerically. In this way, ABHs of different sizes and locations can be easily considered in a plate (the reader is referred to Section 2.3.2 in [27] for details.

The ABH plate modes can be found as follows. Applying the Euler-Lagrange equations in the absence of external work $\left(\partial_{t}\left(\partial_{\dot{\boldsymbol{a}}} \mathscr{L}\right)-\partial_{\boldsymbol{a}} \mathscr{L}=0\right)$ to Eq. (3) results in the generalized eigenvalue problem,

$$
\left(-\omega^{2} \boldsymbol{M}+\boldsymbol{K}\right) \hat{\boldsymbol{A}}=\mathbf{0} .
$$

To solve the eigenproblem in Eq. (8), the normal (real) mode method is employed by solely considering the real part of the stiffness matrix. The $q$-th eigenpair $\left(\lambda_{q}, \hat{\boldsymbol{A}}_{q}\right)$ consists of the eigenvalue $\lambda_{q}=\omega_{q}^{2}$ and the eigenvector $\hat{\boldsymbol{A}}_{q}$. The $q$-th ABH modal shape is recovered from $w_{q}=\hat{\boldsymbol{A}}_{q}^{\top} \boldsymbol{\chi}$, and can be normalized to unit modal mass taking $\widetilde{w}_{q}=\widetilde{\boldsymbol{A}}_{q}^{\top} \boldsymbol{\chi}$, with $\widetilde{\boldsymbol{A}}_{q}=\hat{\boldsymbol{A}}_{q} / \sqrt{N_{q}}$ being the normalized coefficient vector and 

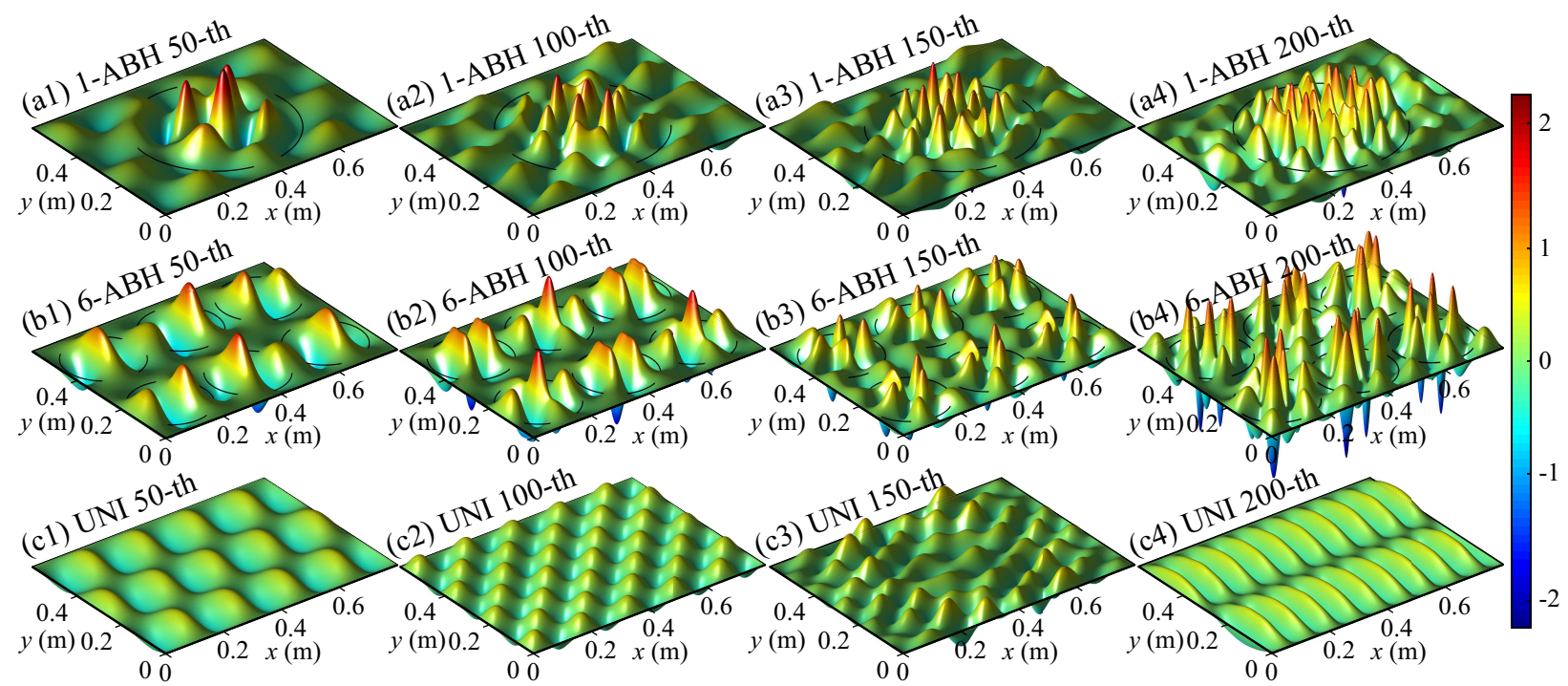

Figure 3: Modal shapes normalized to unit mass for the 1-ABH plate (a1)-(a4), the 6-ABH plate (b1)-(b4) and the reference uniform plate having the same damping layer configuration as the 1-ABH plate (c1)-(c4).

$N_{q}=\hat{\boldsymbol{A}}_{q}^{\top} \boldsymbol{M} \hat{\boldsymbol{A}}_{q}$ the normalization factor. The modal matrix then becomes the identity matrix and the modal stiffness matrix contains the real problem eigenvalues. Damping is included by assuming a diagonal form for the imaginary stiffness matrix containing the modal loss factors, which guarantees the eigenmodes' orthogonality. Although this simple approach has been used in previous works on SmEdA [45] as well as in many other articles on the modal strain energy method (see e.g., [53-56]), it will be worth exploring in the future to which extent this assumption remains valid for the problem at hand. Yet, this is deemed out of the scope of the current study.

Hereafter, subscript $q$ is used to identify individual ABH plate modes belonging to the whole set $\hat{Q}^{\mathrm{R}}$ of ABH plate modes in the analyzed $1 / 3$ octave frequency band, i.e., $q \in \hat{Q}^{\mathrm{R}}$ (see Table. 2 to see how their number increase with frequency). Superscript $\mathrm{R}$ of $\hat{Q}$ refers to the fact that modes in the frequency band are resonant and are to be distinguished from those in the set of non-resonant modes, $\hat{Q}^{\mathrm{NR}}$, which do not belong to the $1 / 3$ octave band but should be also considered in the SmEdA modeling of the Cavity 1 - ABH plate - Cavity 2 system (see [50]). This point will be made more clear in forthcoming sections.

To improve understanding by means of an example, we next calculate some modal frequencies and shapes for the $1-\mathrm{ABH}$ and $6-\mathrm{ABH}$ plates sketched in Fig. 1, and for a reference plate of constant thickness. The

\begin{tabular}{cc}
\hline Geometry parameters & Material parameters \\
\hline$m=2.5$ & $\rho=7800 \mathrm{~kg} / \mathrm{m}^{3}$ \\
$L_{x}=0.8 \mathrm{~m}$ & $E=210 \mathrm{GPa}$ \\
$L_{y}=0.6 \mathrm{~m}$ & $\eta=0.01$ \\
$h_{\mathrm{uni}}=0.005 \mathrm{~m}$ & $\nu=0.3$ \\
$r_{\mathrm{abh} 1}=0.24 \mathrm{~m}, r_{\mathrm{abh} 6}=0.12 \mathrm{~m}$ & \\
$\varepsilon_{1}=0.1595 \mathrm{~m}^{-2.5}, \varepsilon_{6}=0.9021 \mathrm{~m}^{-2.5}$ & $\rho_{v}=950 \mathrm{~kg} / \mathrm{m}^{3}$ \\
$h_{r}=0.0005 \mathrm{~m}$ & $E_{v}=5 \mathrm{GPa}$ \\
$r_{v 1}=0.24 \mathrm{~m}, r_{v 6}=0.12 \mathrm{~m}$ & $\eta_{v}=0.5$ \\
$h_{v}=0.002 \mathrm{~m}$ & $\nu_{v}=0.3$ \\
\hline
\end{tabular}

Table 1: Geometry and material parameters of the ABH plates. $\rho$ : plate density, $\rho_{v}$ : damping layer density, $E$ : plate Young modulus, $E_{v}$ : damping layer Young modulus, $\eta$ : plate loss factor, $\eta_{v}$ : damping layer loss factor, $\nu$ : plate Poisson ratio, $\nu_{v}$ : damping layer Poisson ratio. 
geometrical and material details of the plates are listed in Table. 1 . In the GEM model, we take $m_{x}=m_{y}=6$ to guarantee accurate results, which results in $n_{x}^{i} \in[-4,56]$ and $n_{y}^{i} \in[-4,43]$. This leads to a shape function vector of dimensions $\chi_{2928 \times 1}$ and to mass and stiffness matrices $\boldsymbol{M}_{2928 \times 2928}$ and $\boldsymbol{K}_{2928 \times 2928}$. Likewise, we consider the plate to be simply supported by assigning $k_{i}=1 \times 10^{12} \mathrm{~N} / \mathrm{m}^{2}$ and $p_{i}=0(i=1 \ldots 4)$ to its boundaries.

To begin with, the first 200 modal frequencies of the three plates have been calculated and plotted in Fig. 2a. As one could expect, the inclusion of ABHs diminishes the modal frequencies because the indentations lower the plate structural stiffness. The figure also reflects the 6 -ABH plate has less structural rigidity than the $1-\mathrm{ABH}$ plate. Likewise, the plate modal loss factors are plotted in Fig. $2 \mathrm{~b}$, showing that the potential for energy dissipation is much higher in $\mathrm{ABH}$ plates than in uniform ones having the same amount of damping. On the other hand, note that the modal loss factor maximum values are similar for the $1-\mathrm{ABH}$ and 6 - $\mathrm{ABH}$ plates, but the second show less dispersion. For illustrative purposes, some arbitrary modal shapes of the three plates are presented in Fig. 3 (in particular the 50-th, 100-th, 150-th and 200-th modes). The figure shows how the higher modal vibration amplitude concentrates at the ABH central areas where it can be dissipated by the viscous damping layer. This is in contrast with the standard modal shape patterns for the uniform plate depicted in the third row of Fig. 3. It is observed, though, that for some specific modes (e.g., 150-th) the modal shape differs from that of a non-damped uniform plate. One should have in mind that the plate in the third row of the figure has attached the same circular damping layer used in the 1-ABH plate for a fair comparison. It has been tested, though not reproduced herein, that this damping layer can affect the pattern of some modes, depending on the location of their vibration peaks. When thinning the thickness of the damping layer the 150-th order of a non-damped uniform plate is recovered.

\subsection{Cavity acoustic modes}

The computation of the acoustic modes from a rectangular cavity with rigid boundaries (blocked modes in the SmEdA model) is very standard and analytical expressions can be found for them in many reference textbooks (see e.g., [57]). The modes are obtained from the solution of the Helmholtz equation for the acoustic pressure $\hat{p}(\boldsymbol{x}, \omega)$,

$$
\nabla^{2} \hat{p}-k^{2} \hat{p}=0
$$

(where $k=\omega / c_{0}$ is the acoustic wavenumber and $c_{0}$ the speed of sound in air), supplemented with boundary conditions $\nabla \hat{p} \cdot \boldsymbol{n}=0$ on each boundary surface ( $\boldsymbol{n}$ stands for the outward pointing normal vector).

The modal frequencies of the rectangular cavities 1 and 2 with rigid walls are given by

$$
\begin{aligned}
& \omega_{p}=\frac{c_{0}}{2} \sqrt{\left(\frac{p_{x}}{L_{x}}\right)^{2}+\left(\frac{p_{y}}{L_{y}}\right)^{2}+\left(\frac{p_{z}}{L_{z 1}}\right)^{2}}, \\
& \omega_{r}=\frac{c_{0}}{2} \sqrt{\left(\frac{r_{x}}{L_{x}}\right)^{2}+\left(\frac{r_{y}}{L_{y}}\right)^{2}+\left(\frac{r_{z}}{L_{z 2}}\right)^{2}} .
\end{aligned}
$$

From now on, subscripts $p \in \hat{P}$ and $r \in \hat{R}$ are respectively used to refer to Cavity 1 and Cavity 2 modes, $\hat{P}$ and $\hat{R}$ denoting the corresponding sets of modes in the considered $1 / 3$ octave frequency band. $p_{m}$ and $r_{m}$ with $m=x, y, z$ in Eq. (10) are integer indices to sort the modal frequencies. The number of cavity and $\mathrm{ABH}$ plate modes in a band rapidly increases with frequency as shown in Table. 2. On the other hand, the cavity acoustic modes have the expressions

$$
\begin{aligned}
& p_{p}=\cos \left(\frac{p_{x} \pi x}{L_{x}}\right) \cos \left(\frac{p_{y} \pi y}{L_{y}}\right) \cos \left(\frac{p_{z} \pi z}{L_{z 1}}\right), \\
& p_{r}=\cos \left(\frac{r_{x} \pi x}{L_{x}}\right) \cos \left(\frac{r_{y} \pi y}{L_{y}}\right) \cos \left(\frac{r_{z} \pi z}{L_{z 2}}\right) .
\end{aligned}
$$

For convenience, they are normalized to unit modal stiffness to get

$$
\tilde{p}_{p}=p_{p} / \sqrt{N_{p}} \text { and } \tilde{p}_{r}=p_{r} / \sqrt{N_{r}},
$$



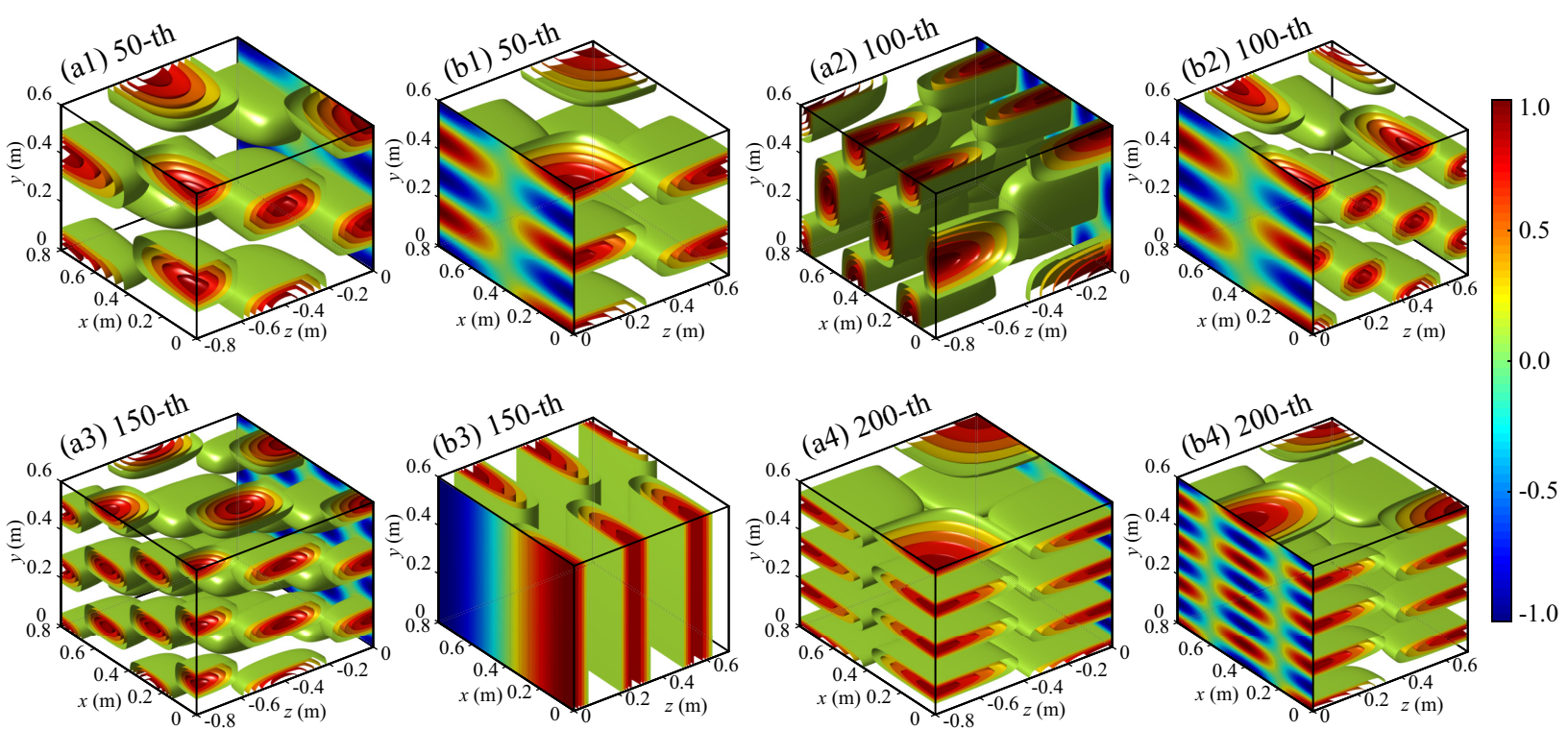

Figure 4: Modal shapes normalized to unit stiffness for Cavity 1 (a1)-(a4) and Cavity 2 (b1)-(b4). The acoustic pressure distribution at the separation wall is also plotted.

with normalization factors

$$
N_{p}=\frac{1}{\rho_{0} c_{0}^{2}} \int_{V_{1}} p_{p}^{2} \mathrm{~d} V \quad \text { and } \quad N_{r}=\frac{1}{\rho_{0} c_{0}^{2}} \int_{V_{2}} p_{r}^{2} \mathrm{~d} V .
$$

The acoustic pressure at points $\boldsymbol{x}_{1}$ and $\boldsymbol{x}_{2}$ inside cavities 1 and 2 under a given source excitation admit modal series expansions,

$$
p\left(\boldsymbol{x}_{1}, t\right)=\sum_{p=1} \phi_{p}(t) \tilde{p}_{p}\left(\boldsymbol{x}_{1}\right) \text { and } \quad p\left(\boldsymbol{x}_{2}, t\right)=\sum_{r=1} \xi_{r}(t) \tilde{p}_{r}\left(\boldsymbol{x}_{2}\right),
$$

with $\phi_{p}(t)$ and $\xi_{r}(t)$ standing for time varying modal amplitudes.

In the present work, we consider Cavity 1 has depth $L_{z 1}=0.8 \mathrm{~m}$ while Cavity 2 has depth $L_{z 2}=0.7$ $\mathrm{m}$. The density of air is chosen to be $\rho_{0}=1.29 \mathrm{~kg} / \mathrm{m}^{3}$, the sound speed $340 \mathrm{~m} / \mathrm{s}$ and the damping loss factor of the two cavities is taken as $\eta_{C 1}=\eta_{C 2}=0.01$. According to Eq. (11) and for illustrative purposes, some normalized acoustic modes of Cavity 1 have been plotted in Figs. 4a1-4a4, and some of Cavity 2 in Figs. 4b1-4b4. As the dimensions of the two cavities are different, one can logically observe in the figure how the same acoustical mode order leads to different pressure distributions within the cavities. The modal acoustic pressure distribution at the $z=0$ section (i.e. on the separating wall) exerted by each cavity has also been represented to highlight the importance of spatial matching, which leads to the need of including

\begin{tabular}{cccccccccccccc}
\hline$f_{c}(\mathrm{~Hz})$ & 400 & 500 & 630 & 800 & 1000 & 1250 & 1600 & 2000 & 2500 & 3150 & 4000 & 5000 & 6300 \\
\hline$\hat{P}$ & 5 & 6 & 12 & 22 & 41 & 71 & 149 & 262 & 519 & 1021 & 1987 & 3893 & 7646 \\
$\hat{Q}^{\mathrm{NR}}$ & 12 & 14 & 19 & 26 & 33 & 42 & 56 & 70 & 88 & 114 & 145 & 183 & 234 \\
$\hat{Q}^{\mathrm{R}}$ & 2 & 5 & 7 & 7 & 9 & 14 & 14 & 18 & 26 & 31 & 38 & 51 & 65 \\
$\hat{R}$ & 4 & 4 & 12 & 21 & 32 & 67 & 126 & 232 & 465 & 896 & 1732 & 3407 & 6711 \\
\hline
\end{tabular}

Table 2: Number of modes in each $1 / 3$ octave band with central frequency $f_{c} . P$ stands for the modes of Cavity $1, Q^{\text {NR }}$ denotes the non-resonant modes of the $1 \mathrm{ABH}$ plate, whereas $Q^{\mathrm{R}}$ indicates the resonant ones, and $R$ implies the modes of Cavity 2. 
non-resonant plate modes for a proper description of energy transmission between cavities. In theory, cavity and plate modes within the considered 1/3 octave frequency band (i.e., resonant modes) should suffice for the analysis. In practice, however, it happens that the pattern of some resonant acoustic modes at $z=0$ matches with the displacement pattern of plate modes outside the frequency band, which get excited and transmit energy between cavities. Such plate modes are referred to as non-resonant modes. It was shown in [50] that for the cavity-panel-cavity problem non-resonant plate modes essentially have frequencies lower than that of the analyzed frequency band and are mostly important below the critical frequency of the panel. The energy contribution from plate modes with higher frequencies than those of the band can be discarded. For instance, the $1 / 3$ octave band with central frequency $f_{\text {cen }}=1000 \mathrm{~Hz}$ has a lower band edge frequency of $f_{\text {low }}=891 \mathrm{~Hz}$ and an upper one of $f_{\text {up }}=1122 \mathrm{~Hz}$. All plate modes with modal frequencies lower than 891 $\mathrm{Hz}$ are considered as non-resonant modes for the $1000 \mathrm{~Hz} 1 / 3$ octave band. There exist 33 of them as shown in Table. 2. The plate modes between $891 \mathrm{~Hz}$ and $1122 \mathrm{~Hz}$ correspond to resonant modes and we have 9 of them (see Table. 2). As said, modes with frequencies larger than $1122 \mathrm{~Hz}$ have negligible influence on the energy transmission at the $1000 \mathrm{~Hz} 1 / 3$ octave band and are ignored. Therefore, the higher the analyzed frequency band the higher the number of non-resonant modes, as observed from the second row of Table. 2.

\subsection{SmEdA model for resonant and non-resonant transmission}

To facilitate comprehension, in what follows the key SmEdA equations will be succinctly presented in a constructive manner (the reader is refereed to $[31,50]$ for full derivations and explanations). Let us first focus on the power balance for an arbitrary mode $p$ of Cavity 1 , belonging to a given $1 / 3$ octave frequency band. At equilibrium, the power $\Pi_{\mathrm{inj}}^{p}$ injected to $p$ via the sound source $Q_{p}$ must equal the power dissipated by the internal damping of Cavity 1, plus that transmitted to the separating ABH plate, plus that conducted to Cavity 2 through non-resonant paths. Mathematically, this can be written as

$$
\Pi_{\mathrm{inj}}^{p}=\Pi_{\mathrm{diss}}^{p}+\sum_{q \in \hat{Q}^{\mathrm{R}}} \Pi_{p q}+\sum_{r \in \hat{R}} \Pi_{p r}, \forall p \in \hat{P},
$$

where $\Pi_{p q}$ represents the time averaged power exchanged between mode $p$ and the resonant plate mode $q$ in the frequency band of interest, and $\Pi_{p r}$ denotes the power from $p$ to resonant mode $r$ in Cavity 2, through a non-resonant mode of the plate.

Expressions for the different terms in Eq. (15) are next provided. The injected power can be computed as,

$$
\Pi_{\mathrm{inj}}^{p}=\frac{\pi}{4} \bar{S}_{Q_{p}}
$$

where

$$
\bar{S}_{Q_{p}}=\left[\frac{\cos \left(\frac{p_{x} \pi x_{s}}{L_{x}}\right) \cos \left(\frac{p_{y} \pi y_{s}}{L_{y}}\right) \cos \left(\frac{p_{z} \pi z_{s}}{L_{z 1}}\right)}{\sqrt{N_{p}}}\right]^{2},
$$

is the power spectral density of the generalized source strength. Likewise, the power of mode $p$ dissipated in Cavity 1 is related to its total energy through,

$$
\Pi_{\mathrm{diss}}^{p}=\omega_{p} \eta_{p} E_{p}
$$

where $\omega_{p}$ and $\eta_{p}$ respectively stand for the modal frequency and the modal loss factor, and $E_{p}$ is the time averaged energy of mode $p$.

Next, we shall determine the power $\Pi_{p q}$ interchanged by mode $p$ of Cavity 1 and mode $q$ of the ABH plate. In the time domain, the governing equations for the dynamics of interacting modes $p$ and $q$ read (see $[50])$,

$$
\begin{aligned}
& \ddot{\varphi}_{p}+\omega_{p} \eta_{p} \dot{\varphi}_{p}+\omega_{p}^{2} \varphi_{p}-W_{p q} \dot{\chi}_{q}=L_{p-q}, \\
& \ddot{\chi}_{q}+\omega_{q} \eta_{q} \dot{\chi}_{q}+\omega_{q}^{2} \chi_{q}-W_{p q} \dot{\varphi}_{p}=L_{q-p},
\end{aligned}
$$


where the time derivative of $\varphi_{p}$ is identified with the modal amplitude $\phi_{p}$ in the modal expansion for the acoustic pressure of Cavity 1 in Eq. (14) (i.e., $\dot{\varphi}_{p} \equiv \phi_{p}$ ), and

$$
\begin{aligned}
W_{p q} & =\int_{S} \widetilde{w}_{q} \widetilde{p}_{p} \mathrm{~d} S=\int_{0}^{L_{y}} \int_{0}^{L_{x}}\left(\widetilde{\boldsymbol{A}}_{q}^{\top} \boldsymbol{\chi}\right)\left[\cos \left(\frac{p_{x} \pi x}{L_{x}}\right) \cos \left(\frac{p_{y} \pi y}{L_{y}}\right) / \sqrt{N_{p}}\right] \mathrm{d} x \mathrm{~d} y \\
& =\frac{\widetilde{\boldsymbol{A}}_{a}^{\top}}{\sqrt{N_{p}}}\left\{\left[\int_{0}^{L_{x}} \boldsymbol{\alpha}_{x} \cos \left(\frac{p_{x} \pi x}{L_{x}}\right) \mathrm{d} x\right] \otimes\left[\int_{0}^{L_{y}} \boldsymbol{\alpha}_{y} \cos \left(\frac{p_{y} \pi y}{L_{y}}\right) \mathrm{d} y\right]\right\},
\end{aligned}
$$

is the modal interaction work between mode $p$ of Cavity 1 and plate mode $q$ at the interface $z=0$. The right hand side terms in Eqs. (19a) and (19b) are given by

$$
\begin{aligned}
L_{p-q} & =Q_{p}+\sum_{\substack{\tilde{q} \in \hat{Q}^{\mathrm{R}} \\
\widetilde{q} \neq q}} W_{p \widetilde{q}} \dot{\chi}_{\widetilde{q}}+\sum_{r \in \hat{R}}\left(\sum_{q \in \hat{Q}^{\mathrm{NR}}} W_{p q} W_{r q}\right) \chi_{r}, \\
L_{q-p} & =\sum_{\substack{\widetilde{\widetilde{p}} \in \hat{P} \\
\tilde{p} \neq p}} W_{\widetilde{p} q} \dot{\varphi}+\sum_{r \in \hat{R}} W_{r q} \dot{\zeta}_{r} .
\end{aligned}
$$

$L_{p-q}$ in Eq. (21a) accounts for the influence of all resonant plate modes but $q$ on $p$ (i.e. all $\tilde{q} \in \hat{Q}^{\mathrm{R}}$ with $\tilde{q} \neq q$ ) and for the influence of all resonant modes $r \in \hat{R}$ in Cavity 2, which affect $p$ via the plate non-resonant modes $q \in \hat{Q}^{\mathrm{NR}}$. Similarly, $L_{q-p}$ in Eq. (21b) describes the effects of all Cavity 1 resonant modes but $p$ on the plate resonant mode $q$, as well as the effects of all resonant modes $r$ from Cavity 2 . The quantity $\zeta_{r}$ in the second summation of $L_{q-p}$ corresponds to $\dot{\zeta}_{r} \equiv \xi_{r}$, with $\xi_{r}$ being the modal amplitude of mode $r$ of Cavity 2 in Eq. (14).

If, as usual, one assumes that $L_{p-q}$ and $L_{q-p}$ are uncorrelated white noise forces, the modal interaction between Cavity 1 mode $p$ and resonant plate mode $q$ can be approximated through gyroscopic coupling, which results in the coupling power,

$$
\Pi_{p q} \approx \beta_{p q}\left(E_{p}-E_{q}\right)
$$

where

$$
\beta_{p q}=W_{p q}^{2} \frac{\omega_{p} \eta_{p} \omega_{q}^{2}+\omega_{q} \eta_{q} \omega_{p}^{2}}{\left(\omega_{p}^{2}-\omega_{q}^{2}\right)^{2}+\left(\omega_{p} \eta_{p}+\omega_{q} \eta_{q}\right)\left(\omega_{p} \eta_{p} \omega_{q}^{2}+\omega_{q} \eta_{q} \omega_{p}^{2}\right)},
$$

is the modal coupling factor.

Proceeding analogously, one can obtain the power between resonant plate mode $q$ and Cavity 2 mode $r$ as,

$$
\Pi_{q r} \approx \beta_{q r}\left(E_{q}-E_{r}\right)
$$

with modal coupling factor

$$
\beta_{q r}=W_{q r}^{2} \frac{\omega_{q} \eta_{q} \omega_{r}^{2}+\omega_{r} \eta_{r} \omega_{q}^{2}}{\left(\omega_{q}^{2}-\omega_{r}^{2}\right)^{2}+\left(\omega_{q} \eta_{q}+\omega_{r} \eta_{r}\right)\left(\omega_{q} \eta_{q} \omega_{r}^{2}+\omega_{r} \eta_{r} \omega_{q}^{2}\right)} .
$$

The modal interaction work in Eq. (25) is now given by,

$$
W_{q r}=\int_{S} \widetilde{w}_{q} \widetilde{p}_{r} \mathrm{~d} S=\frac{\widetilde{\boldsymbol{A}}_{q}^{\top}}{\sqrt{N_{r}}}\left\{\left[\int_{0}^{L_{x}} \boldsymbol{\alpha}_{x} \cos \left(\frac{r_{x} \pi x}{L_{x}}\right) \mathrm{d} x\right] \otimes\left[\int_{0}^{L_{y}} \boldsymbol{\alpha}_{y} \cos \left(\frac{r_{y} \pi y}{L_{y}}\right) \mathrm{d} y\right]\right\} .
$$



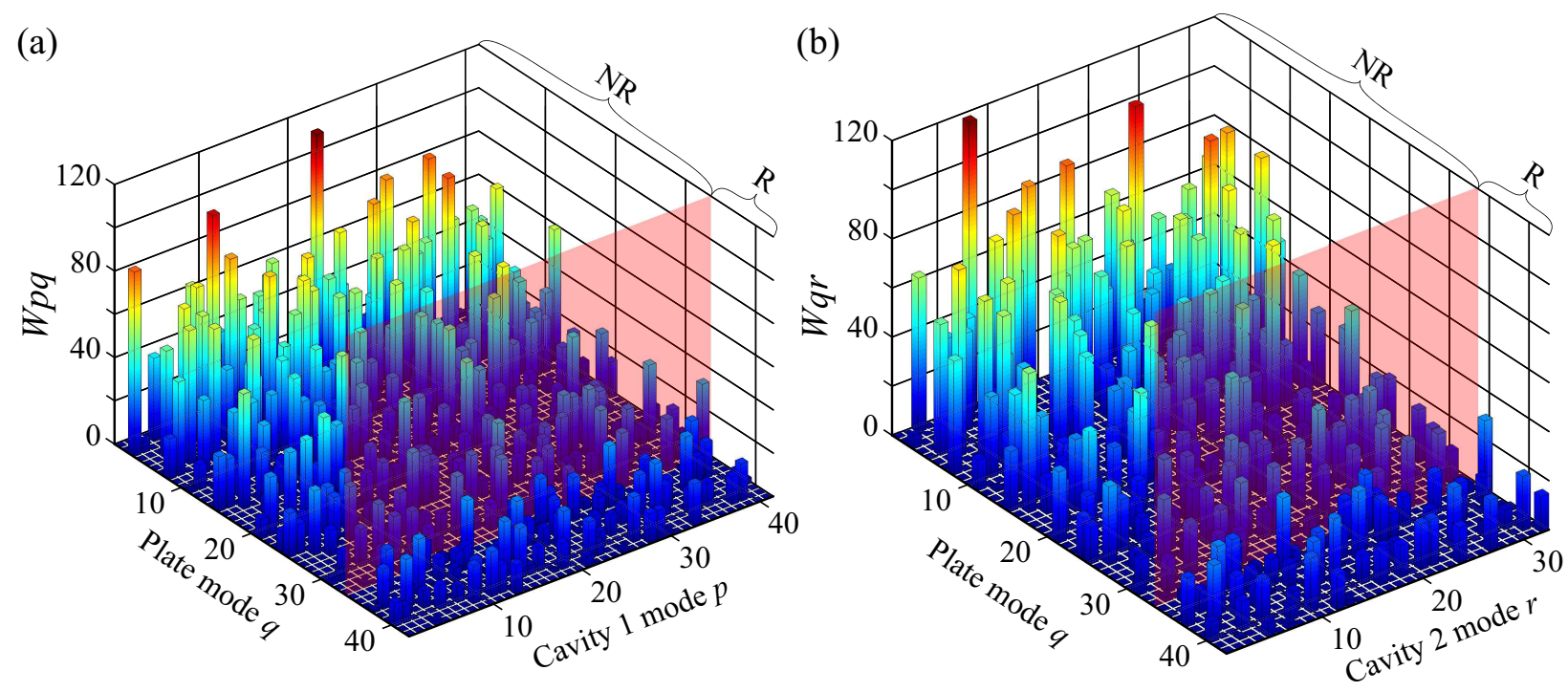

Figure 5: Modal interaction work (absolute value) of (a) 1-ABH plate and Cavity 1, (b) 1-ABH plate and Cavity 2, at the $1 / 3$ octave frequency band of $f_{c}=1000 \mathrm{~Hz}$. The vertical pink panels at $p=33$ indicates the border between resonant $(\mathrm{R})$ and non-resonant $(\mathrm{NR})$ plate modes.

In what concerns non-resonant transmission between Cavity 1 mode $p$ and Cavity 2 mode $r$, this is characterized by spring coupling [50]. The interaction power can be approximated once more as,

$$
\Pi_{p r}=\beta_{p r}\left(E_{p}-E_{r}\right),
$$

where now

$$
\beta_{p r}=\left(\sum_{q \in \hat{Q}^{\mathrm{NR}}} W_{p q} W_{r q}\right)^{2} \frac{\omega_{p} \eta_{p}+\omega_{r} \eta_{r}}{\left(\omega_{p}^{2}-\omega_{r}^{2}\right)^{2}+\left(\omega_{p} \eta_{p}+\omega_{r} \eta_{r}\right)\left(\omega_{p} \eta_{p} \omega_{r}^{2}+\omega_{r} \eta_{r} \omega_{p}^{2}\right)}
$$

The modal interaction works $W_{p q}$ at the $1 / 3$ octave band with central frequency $f_{c}=1000 \mathrm{~Hz}$, between all resonant and non-resonant $1 \mathrm{ABH}$ plate modes $q \in \hat{Q}^{\mathrm{R}} \cup \hat{Q}^{\mathrm{NR}}$ and all Cavity 1 modes $p \in \hat{P}$ have been plotted in Fig. 5a, while the works, $W_{p q}$, for the plate modes $q \in \hat{Q}^{\mathrm{R}} \cup \hat{Q}^{\mathrm{NR}}$ and Cavity 2 modes $r \in \hat{R}$ are presented in Fig. 5b. As observed in the figures, interaction works involving non-resonant modes can be very large, which means that if spatial matching occurs between plate non-resonant modes and acoustic cavity modes, neglecting the former may result in strong errors in the prediction of energy transmission between cavities [50, 51].

Once known how to determine the modal coupling loss factors between all types of modes, we can establish the power balance equations between modes in the three subsystem model Cavity 1 - ABH plate - Cavity 2. Writing equivalent equations to Eq. (15) for the ABH plate and Cavity 2, and making use of Eqs.(22), (24) and (27), we arrive at,

$$
\begin{aligned}
& \omega_{p} \eta_{p} E_{p}+\sum_{q \in \hat{Q}^{\mathrm{R}}} \beta_{p q}\left(E_{p}-E_{q}\right)+\sum_{r \in \hat{R}} \beta_{p r}\left(E_{p}-E_{r}\right)=\Pi_{\mathrm{inj}}^{p}, \forall p \in \hat{P}, \\
& \sum_{p \in \hat{P}} \beta_{p q}\left(E_{q}-E_{p}\right)+\omega_{q} \eta_{q} E_{q}+\sum_{r \in \hat{R}} \beta_{q r}\left(E_{q}-E_{r}\right)=0, \forall q \in \hat{Q}^{\mathrm{R}}, \\
& \sum_{p \in \hat{P}} \beta_{p r}\left(E_{r}-E_{p}\right)+\sum_{q \in \hat{Q}^{\mathrm{R}}} \beta_{q r}\left(E_{r}-E_{q}\right)+\omega_{r} \eta_{r} E_{r}=0, \forall r \in \hat{R} .
\end{aligned}
$$


Eq. (29) can be compacted in matrix form by means of block matrices $\boldsymbol{\beta}_{i j}(i, j=1 \ldots 3)$,

$$
\left[\begin{array}{ccc}
\boldsymbol{\beta}_{11} & \boldsymbol{\beta}_{12} & \boldsymbol{\beta}_{13} \\
\boldsymbol{\beta}_{12}^{\top} & \boldsymbol{\beta}_{22} & \boldsymbol{\beta}_{23} \\
\boldsymbol{\beta}_{13}^{\top} & \boldsymbol{\beta}_{23}^{\top} & \boldsymbol{\beta}_{33}
\end{array}\right]\left[\begin{array}{c}
\boldsymbol{E}_{1} \\
\boldsymbol{E}_{2} \\
\boldsymbol{E}_{3}
\end{array}\right]=\left[\begin{array}{c}
\boldsymbol{\Pi}_{1} \\
\mathbf{0} \\
\mathbf{0}
\end{array}\right],
$$

where $\boldsymbol{E}_{i}(i=1 \ldots 3)$ are modal energy vectors for each subsystem and $\boldsymbol{\Pi}_{1}$ is the external modal power vector injected in Cavity 1. Eq. (30) can be rewritten as

$$
\beta E=\Pi .
$$

with obvious identification of terms. Solving the system of equations in Eq. (31) provides the energies of all modes in the three subsystems. The entries of the block matrices in Eq. (30) are given in Appendix A for completeness.

\subsection{Energy noise reduction and transmission loss between cavities}

The transmission loss of the ABH plates can be obtained quite straightforwardly once we know the modal energies of the cavities from the solution to Eq. (30). The total energies of Cavity 1 and Cavity 2 result from the summation of the energies of their respective modes in the considered $1 / 3$ octave band, i.e,

$$
E_{C 1}=\sum_{p \in \hat{P}} E_{p} \text { and } E_{C 2}=\sum_{r \in \hat{R}} E_{r} .
$$

The energy noise reduction (ENR) between cavities can then be defined as

$$
\mathrm{ENR}=10 \log _{10}\left(\frac{E_{C 1}}{E_{C 2}}\right),
$$

which is related to the standard transmission loss (TL) by,

$$
\mathrm{TL}=\mathrm{ENR}-10 \log _{10}\left(\frac{V_{1}}{V_{2}}\right)-10 \log _{10}\left(\frac{4 \eta_{C 2} \omega_{c} V_{2}}{c_{0} L_{x} L_{y}}\right) .
$$

Note that the TL and ENR levels only differ by a constant value at each $1 / 3$ octave band of central angular frequency $\omega_{c}$. As seen from Eq. (34), the difference involves some geometrical parameters like the volumes of the cavities $V_{1}$ and $V_{2}$, or the surface $L_{x} L_{y}$ of the separating $\mathrm{ABH}$ plate, and some physical ones like the speed of sound and the loss factor of Cavity 2. Given that one can easily switch between TL and ENR, in the following section we will basically present ENR results as these directly stem from SmEdA models. Also, this eases comparison with previous works on transmission between cavities using SmEdA (see e.g., [50, 51]).

\section{Numerical results}

\subsection{Resonant and non-resonant transmission between cavities}

Before start analyzing the potential of embedding ABHs on plates to increase the ENR (read also $\mathrm{TL}$ ) between cavities, let us present some simulations showing the importance of considering non-resonant transmission in the simulations. That is specially significant below the critical frequency of plates, which for the uniform one in forthcoming examples is $f_{\text {crit }}=2300 \mathrm{~Hz}$. It should be remarked that the notion of critical frequency does not directly apply to $\mathrm{ABH}$ plates due to the change in wave propagation speed because of the power-law thickness variation. Nonetheless, including non-resonant transmission is also mandatory for $\mathrm{ABH}$ plates at low frequencies, as it will be next shown.

Consider a 1-ABH plate (see Table 1) separating Cavity 1 and Cavity 2, and a monopole source in the first one located at $\boldsymbol{x}_{s}=\left(x_{s}, y_{s}, z_{s}\right)=(0.24,0.42,-0.26) \mathrm{m}$. We solve Eq. (31) for the $1 / 3$ octave band centered at $f_{c}=1000 \mathrm{~Hz}$ taking into acount both resonant and non-resonant transmission. The solution 


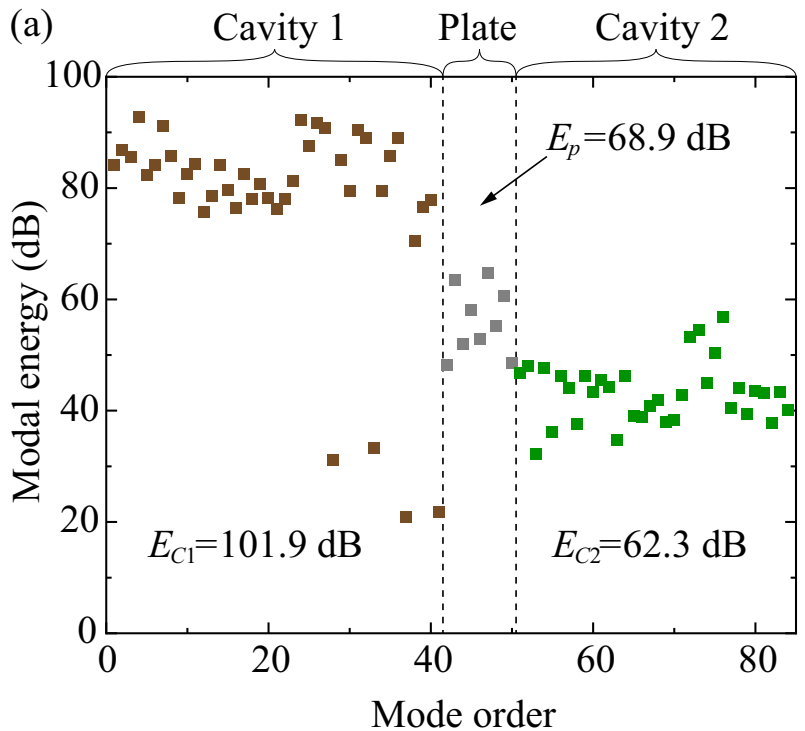

(b) Cavity $1 \quad$ Plate Cavity 2

(c)

Cavity 2


(d) Cavity $1 \quad$ Cavity 2

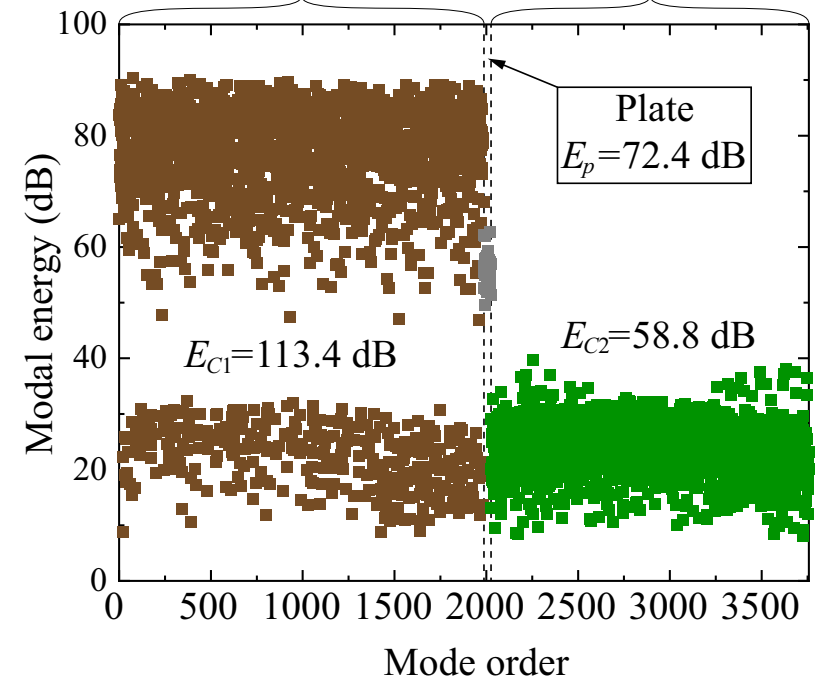

Figure 6: (a) Modal energies of Cavity 1, 1-ABH plate and Cavity 2 considering resonant and non-resonant transmission at the $1 / 3$ octave band of $f_{c}=1000 \mathrm{~Hz}$. (b) Modal energies of Cavity 1, 1-ABH plate and Cavity 2 only considering resonant transmission at the $1 / 3$ octave band of $f_{c}=1000 \mathrm{~Hz}$. (c) Modal energies of Cavity 1, 1-ABH plate and Cavity 2 considering resonant and non-resonant transmission at the $1 / 3$ octave band of $f_{c}=4000 \mathrm{~Hz}$. (d) Modal energies of Cavity 1 , 1 -ABH plate and Cavity 2 only considering resonant transmission at the $1 / 3$ octave band of $f_{c}=4000 \mathrm{~Hz}$.

energy vector $\boldsymbol{E}=\left(\begin{array}{lll}\boldsymbol{E}_{1}^{\top} & \boldsymbol{E}_{2}^{\top} & \boldsymbol{E}_{2}^{\top}\end{array}\right)^{\top}$ sequentially contains the modal energies of the Cavity 1, 1-ABH plate and Cavity 2 subsystems. These modal energies have been plotted in Fig. 6a, each brown point denoting the energy of a mode in Cavity 1 in the $1000 \mathrm{~Hz} 1 / 3$ octave band. Analogously, grey points correspond to modal energies of the 1-ABH plate and green points to those in Cavity 2. Note that the modal energies in Cavity 1 are split into two groups, one of them having very low energy. Although not shown in figures, we have checked that these energies correspond to modes such that the source location, $\boldsymbol{x}_{s}$, lies in some of their nodal lines. Consequently, they get barely excited.

The total energies of the cavities obtained from modal energy summation (see Eq. (32)) are also indicated in Fig. 6a, together with that of the plate. The computed values are $E_{C 1}=101.9 \mathrm{~dB}, E_{p}=68.9 \mathrm{~dB}$ and 
$E_{C 2}=62.3 \mathrm{~dB}$, which provide an ENR value at $1000 \mathrm{~Hz}$ of $39.6 \mathrm{~dB}$, see Eq. (33). For comparison, Fig. $6 \mathrm{~b}$ presents the modal energies from Eq. (31) when only resonant transmission is contemplated. As observed, the total energies of Cavity 1 and the 1-ABH plate remain identical, while that of the second Cavity 2 reduces to $52 \mathrm{~dB}$. This yields an ENR of $49.9 \mathrm{~dB}$, which overestimates that in Fig. 6a by $10.3 \mathrm{~dB}$. Neglecting non-resonant transmission can therefore result in strong prediction errors. The latter diminish for higher frequencies. For instance, in Figs. 6c and 6d we depict analogous results to those in Figs. $6 \mathrm{a}$ and $6 \mathrm{~b}$ but for the $4000 \mathrm{~Hz} 1 / 3$ octave band. The ENR at $4000 \mathrm{~Hz}$ when both resonant and non-resonant transmission are included becomes $51.1 \mathrm{~dB}$, while it is $54.6 \mathrm{~dB}$ when non-resonant modes are neglected. The difference between both cases is still meaningful, $3.5 \mathrm{~dB}$, but much smaller than for the $1000 \mathrm{~Hz}$ frequency band. On the other hand, notice that the modal energies in Cavity 1 get also split into two groups in the $4000 \mathrm{~Hz}$ band, the low energy ones corresponding, as said before, to modes whose nodal lines contain the source point. In view of the results of Fig. 6, non-resonant transmission will be included in all subsequent simulations.

To end this section, let us comment that SmEdA constitutes a very fast prediction tool, which makes it very suitable for parametric analysis in the mid-high frequency range. For the problem at hand, it only took $55.27 \mathrm{~s}$ for calculating the ENR of the 13 frequency bands listed in Table. 2, using a vectorized MATLAB code on a personal computer with CPU Intel Core i5-3210M 2.50 GHz and with RAM 16.0 GB. As for the SmEdA computations, it shall be noted that all plate and cavity modes in sections 3.1 and 3.2 , as well as their representations in Figs. 3 and 4 have been also computed using a MATLAB in-house code.

\subsection{Energy noise reduction for a 1-ABH plate. Parametric analysis}

Let us begin investigating the ENR of a plate with a single embedded ABH. Two types of ABHs are tested, the standard circular one and the annular ABH. The latter was proposed in [49] for suppressing vibrations in plates excited at a particular region. The excitation area is surrounded by a ring having an $\mathrm{ABH}$ profile, which diminishes vibration transmission to the rest of the plate. The features of the circular and annular ABHs are provided in Table 1. As shown in Fig. 7a, the ENR of both ABH plates clearly enhance that of a uniform plate beyond the $1000 \mathrm{~Hz} 1 / 3$ octave band. The uniform plate has a critical frequency of $f_{\text {crit }}=2300 \mathrm{~Hz}$ and exhibits a significant ENR drop in the vicinities of it. This is bypassed by the $\mathrm{ABH}$ plates, as predicted by SmEdA and in accordance with the experimental results in [35]. Besides, if we compare the performance of the circular and annular ABH plates, the former performs better at all frequencies. This may be attributed to the fact that the mass of the annular ABH plate is smaller than that of the circular one for the analyzed designs, and mass plays a critical role in augmenting the ENR at low frequencies. If we define the mass reduction ratio as $r_{\Delta m}=\Delta m / m=1-\int_{S} h(x, y) \mathrm{d} S /\left(L_{x} L_{y} h_{\mathrm{uni}}\right)$, it turns out that for the circular ABH we have $r_{\Delta m}=18.85 \%$, while for the annular one $r_{\Delta m}=24.24 \%$. In what follows, only results for circular ABHs will be considered, unless specified.

An ABH indentation helps improving the ENR through a separating plate mainly for two reasons: i) the insertion of an $\mathrm{ABH}$ with a damping layer at its central region strongly reduces the vibrations all over the plate surface; ii) when supersonic flexural waves enter the $\mathrm{ABH}$ their propagating speed slow down and become subsonic at the central region. As a consequence, the radiation efficiency drastically diminishes [18]. Nevertheless, an ABH indentation also has an adverse outcome for ENR because it reduces the mass of the plate. So a proper balance is needed between all these effects and the ABH must be carefully designed. To augment the ENR at low frequencies, in the future one could consider testing the performance of double $\mathrm{ABH}$ panels or the performance of the tunneled double-leaf ABHs in [20] (less mass is removed from the plate with that design and a double side $\mathrm{ABH}$ effect takes place in it).

Next, let us explore how geometrical parameters influence the ENR of a 1-circular ABH plate. Three significant $\mathrm{ABH}$ parameters will be inspected, namely the residual thickness, $h_{r}$, the $\mathrm{ABH}$ radius, $r_{\mathrm{abh}}$, and the $\mathrm{ABH}$ order, $m$. As regards the former and for the sake of convenience, we define the thickness ratio, $t=h_{r} / h_{\text {uni }}$, to study its performance. Results are plotted in Fig. $7 \mathrm{~b}$, for the three different values $t=\{1: 5,1: 10,1: 15\}$. As observed in the figure, the best ENR is achieved for $t=1: 5$ in the most critical frequency range comprising $[800,2500] \mathrm{Hz}$. This might be viewed as counter-intuitive because all studies concerning ABH plate vibrations indicate that the smaller the truncation thickness the better. As remarked before, however, in what concerns ENR diminishing $t$ also implies reducing the plate mass which is detrimental. The mass reduction ratios for $t=1: 5, t=1: 10$, and $t=1: 15$ are, respectively, 

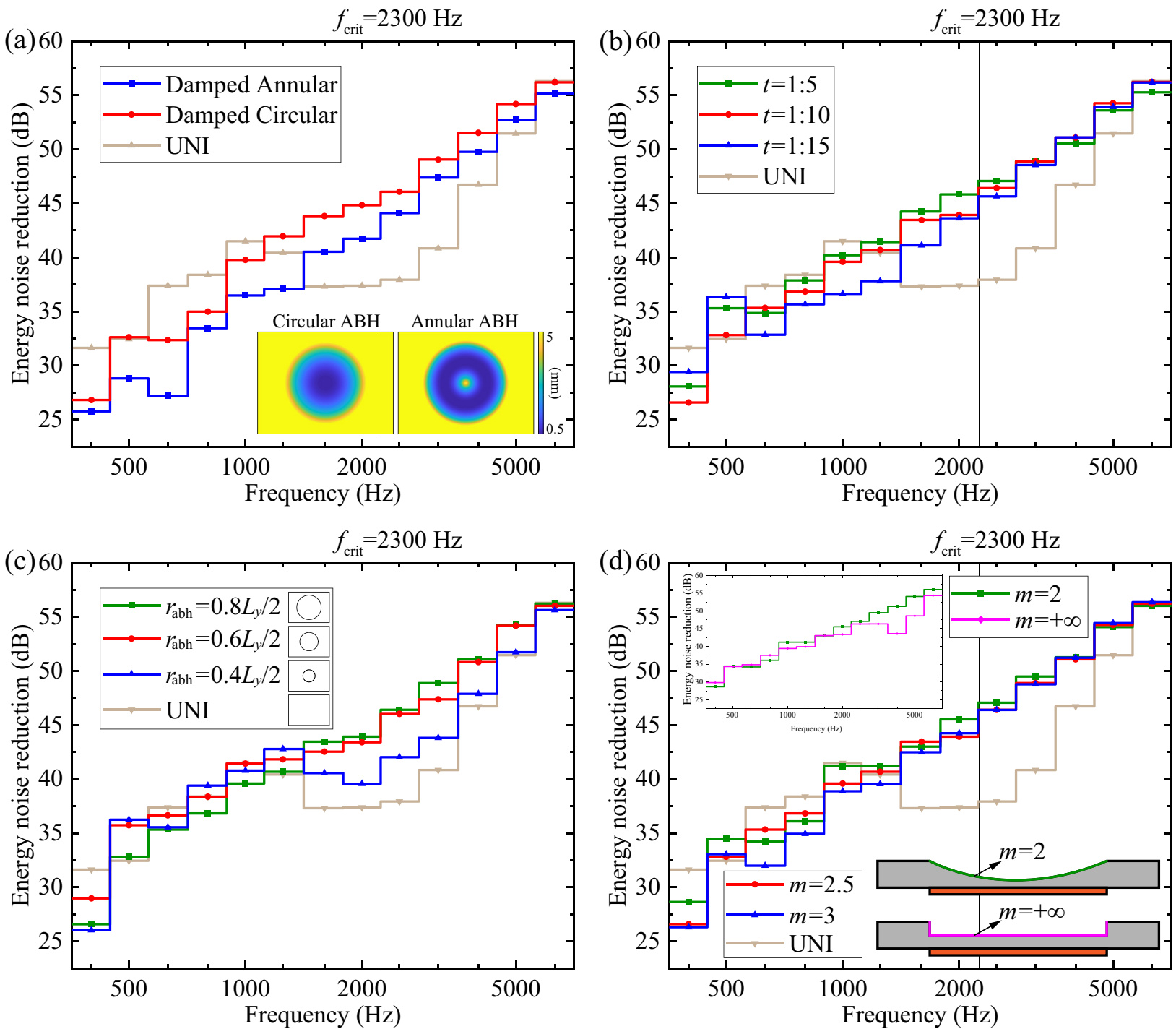

Figure 7: Energy noise reduction (ENR) for 1-ABH plate. (a) Comparison between circular and annular ABHs. (b) Effects of residual thickness. (c) Effects of the ABH radius. (d) Effects of the ABH order (the residual thickness for $m=+\infty$ is $0.0027 \mathrm{~m}$ to equal the $1-\mathrm{ABH}$ plate mass with $m=2) . f_{\text {crit }}=2300 \mathrm{~Hz}$ represents the critical frequency of the uniform plate.

$r_{\Delta m}=16.76 \%, r_{\Delta m}=18.85 \%$, and $r_{\Delta m}=19.55 \%$. For the present example, the best balance in terms of ENR is achieved with $t=1: 5$. This thickness ratio avoids the ENR drop at the critical frequency of the uniform plate and that is still massive enough to perform well at low frequencies (see Fig. 7b).

The second parameter to be analyzed is the $\mathrm{ABH}$ radius and the three values $r_{\mathrm{abh}}=[0.8,0.6,0.4] L_{y} / 2$ are considered for it. It is well known that an ABH starts functioning beyond the so-called diameter cut-on frequency $f_{\text {rabh }}$, which determines that the wavelength of a wave entering the $\mathrm{ABH}$ must be smaller than the ABH diameter. This frequency is given by $f_{\mathrm{rabh}}=\frac{\pi h_{\mathrm{uni}}}{4 r_{\mathrm{abh}}} \sqrt{\frac{E}{3 \rho}}$ and has values $f_{\mathrm{rabh}}=[49,65,98] \mathrm{Hz}$ for the three tested $\mathrm{ABH}$ radii. Nonetheless, $f_{\text {rabh }}$ is not the sole threshold frequency to surpass for an ABH to work properly. Actually, a smoothness condition is required to guarantee a good impedance matching for input waves. If the power-law profile is too steep, waves get reflected and the ABH becomes inefficient. A smoothness cut-on frequency $f_{\varepsilon}=\frac{\varepsilon}{2 \pi} \sqrt{\frac{E}{3 \rho}}$ is then defined with values $f_{\varepsilon}=[76,156,430] \mathrm{Hz}$ for the current 

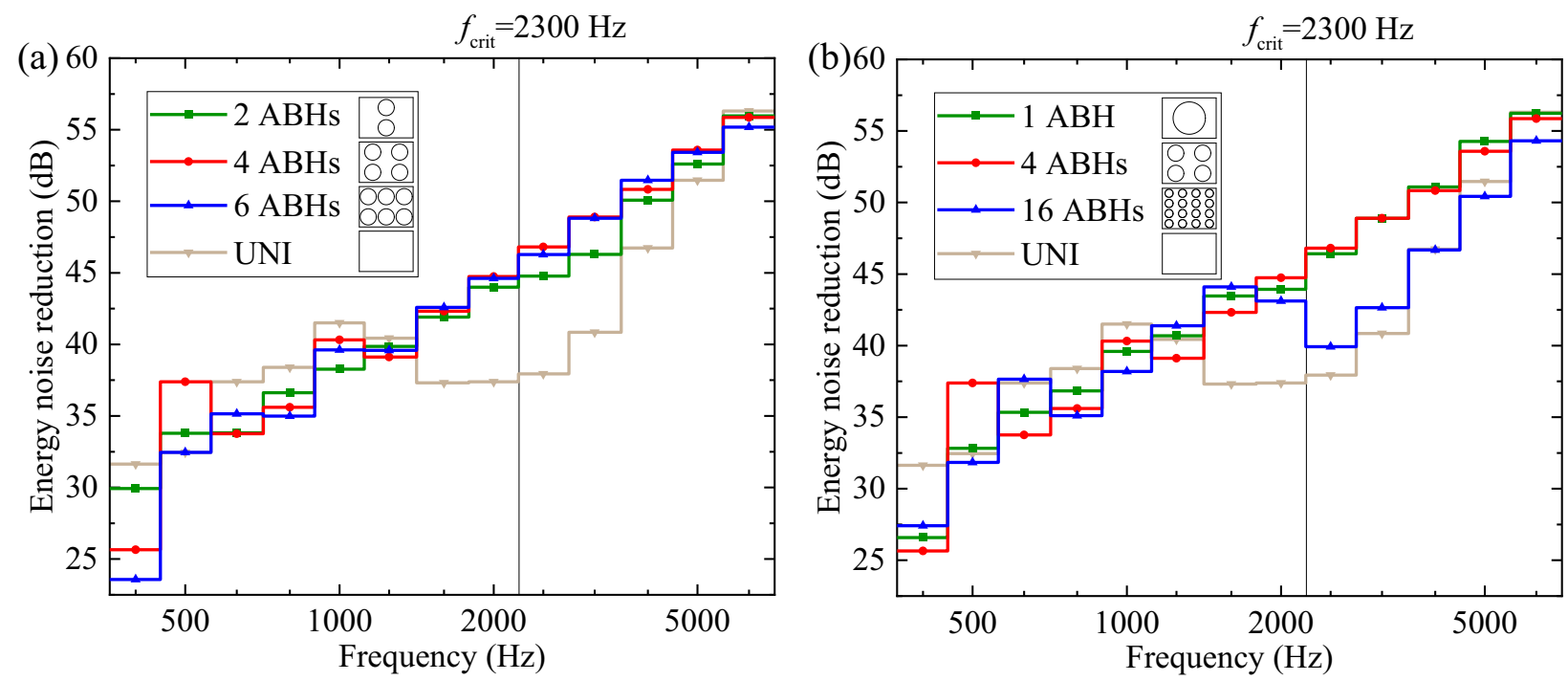

Figure 8: Energy noise reduction (ENR) for plates with multiple ABH indentations. (a) Influence of increasing the number of ABHs. (b) Effects of splitting a big $\mathrm{ABH}$ into smaller ones, while keeping the same total ABH area and mass reduction.

case. Numerical tests have revealed (see e.g., [27]) that ABHs become fully operative for frequencies three times bigger than $f_{\varepsilon}$, i.e., for $f \geq 3 f_{\varepsilon}=[228,468,1290] \mathrm{Hz}$ in our problem. As regards the ENR, again two opposed effects must balance. The larger the $\mathrm{ABH}$ radius the smaller the frequency where it starts functioning, but also the smaller the mass of the plate. The results in Fig. 7c show that the highest ENR values in the range $[1250,5000] \mathrm{Hz}$ are obtained for the largest $\mathrm{ABH}$ radius $r_{\mathrm{abh}}=0.8 L_{y} / 2$. However, if one focus on the $[500,1250] \mathrm{Hz}$ range, it turns out the plate with the largest $\mathrm{ABH}$ is too light and those with smaller ABHs perform better. Likewise, note that the plate with the smallest $\mathrm{ABH}$ radius, $r_{\mathrm{abh}}=0.4 L_{y} / 2$, can hardly compensate the critical frequency drop, despite $f_{\text {crit }}=2300 \mathrm{~Hz}$ surpasses $1290 \mathrm{~Hz}$, which is three times its smoothness cut-on frequency. One may wonder whether embedding multiple ABH indentations on the plate could overcome this problem. This will be investigated in next subsection. Prior to this, however, the effects that the ABH order has on the ENR between cavities will be investigated.

The optimal performance of ABHs is achieved when their order is in the range $m \in[2,3]$. As observed in Fig. 7 d, taking $m=2,2.5$ or 3 yields very similar ENR values in the higher frequency range, $f \geq 4000 \mathrm{~Hz}$. However, $m=2$ works better for lower frequencies because of less mass reduction. To illustrate that eliminating the drop of the ENR at the critical frequency is due to the ABH effect and not to stiffness and/or mass variations associated to indentations, we have included the special case of $m=+\infty$ in the figure. The latter corresponds to the extreme situation in which there is an abrupt transition between the plate uniform thickness and the residual thickness. For this case, the truncation thickness has been chosen as $h_{r}=0.0027 \mathrm{~m}$ to keep the same mass reduction ratio $r_{\Delta m}=18.85 \%$ than for the $\mathrm{ABH}$ of order $m=2$. For $m=+\infty$ no ABH effect is expected, as there is no power-law profile. It can be readily checked from the results in the inner window of Fig. $7 \mathrm{~d}$, that $m=+\infty$ only performs slightly better than $m=2$ for three $1 / 3$ octave bands, namely $f_{c}=[400,630,800] \mathrm{Hz}$, but it is worst for all the others. In particular, the case $m=+\infty$ exhibits a clear ENR drop at the band centered at $f_{c}=4000 \mathrm{~Hz}$, which can be partially attributed to the fact that a uniform plate with thickness $h_{r}=0.0027 \mathrm{~m}$ has a critical frequency of $f_{\text {crit }}=4340 \mathrm{~Hz}$. The comparison between cases $m=2$ and $m=+\infty$ clearly demonstrates that the increase of ENR in plates with embedded $\mathrm{ABH}$ is a consequence of the $\mathrm{ABH}$ effect and cannot be attributed to a mere thickness reduction.

\subsection{Energy noise reduction for a plate with multiple ABHs}

Two types of situations are analyzed to determine the potential benefits of including more than one $\mathrm{ABH}$ in plates to improve the ENR. In the first one we deal with a plate hosting 2, 4, and 6 ABHs. All 
ABHs have the same geometry and therefore the same diameter and smoothness cut-on frequencies, resulting in $3 f_{\varepsilon}=1290 \mathrm{~Hz}$. As seen in Fig. 8a, the plate with $4 \mathrm{ABHs}$ performs better than the 2-ABH plate for $f \in[1250,5000] \mathrm{Hz}$, because it has larger $\mathrm{ABH}$ area (note that the 6 - $\mathrm{ABH}$ plate exhibits very close results to the $4-\mathrm{ABH}$ one in this region). For lower frequencies however, mass becomes the key parameter, as observed in the previous section, and the 2-ABH plate performs better. Whether to chose one ABH plate or another will obviously depend on the particular problem at hand and the need to reduce transmission at a given frequency range, but in general terms the $4-\mathrm{ABH}$ plate offers the most balanced solution for the example in this work.

The second situation consists in keeping the total ABH area and mass reduction constant $\left(r_{\Delta m}=\right.$ $18.85 \%$ ), by splitting a big $\mathrm{ABH}$ into smaller ones. The results are plotted in Fig. 8b, where we consider dividing a single, big $\mathrm{ABH}$, into $4 \mathrm{ABH}$ and then into 16. Three times the smoothness cut-on frequencies of the ABHs, $3 f_{\varepsilon}$, have respective values 228, 1290 and $7299 \mathrm{~Hz}$. One can appreciate in the figure that the 16 -ABH plate has an ENR drop at the band $f_{c}=2500 \mathrm{~Hz}$. The reason for that is the ABH effect is still not functioning properly for this plate because $3 f_{\varepsilon}=7299 \mathrm{~Hz}$ is much bigger than $2500 \mathrm{~Hz}$. In contrast, the other two plates behave very similarly beyond $1600 \mathrm{~Hz}$ and avoid the critical frequency drop of the uniform plate because they have the same total mass and total ABH area, and the ABH is fully operative for them (note that $1600 \mathrm{~Hz}$ is greater than the threshold of $3 f_{\varepsilon}$ for both plates). At lower frequencies, however, the ENR behavior becomes quite complex for the three plates. They all have the same mass so their performance is dictated by the structural stiffness and mass distribution, which makes them have larger or smaller ENR depending on the frequency band.

\subsection{Damping layer influence on the energy noise reduction}

Let us finally focus on studying the influence of the ABH plate damping layer on the ENR between cavities. The case of a plate with a single ABH is addressed. To begin with, Fig. 9a plots the ENR results for a 1-ABH plate and uniform plate, with and without damping. As observed, damping plays an essential role to get reasonable ENR values. It is therefore worth exploring the effects associated to it. We may distinguish three of them: i) added mass, ii) added stiffness and iii) energy dissipation. To evaluate their separate contributions, we can first set $\rho_{v}=0$ to see what happens if there is no added mass, then force $\operatorname{Re}\left(D_{v}\right)=0$ to investigate the impact of neglecting the added stiffness, and finally fix $\eta_{v}=0$ to examine energy dissipation. In Fig. 9b, we show the ENR of the damped (black line) and undamped (grey line) 1-ABH plate. When the added mass is set to zero (red line) the ENR diminishes at all bands with respect to the black line. So the added mass effect of the damping layer is beneficial. The opposite occurs when we set the stiffness to zero. The ENR increases which means that the additional stiffness introduced by the damping layer is detrimental. As one could expect, though, the most important effect is that of energy dissipation. If we set $\eta_{v}=0$ the ENR drops about $10 \mathrm{~dB}$ at most frequency bands.

To end the analysis, we investigate the influence of the radius and thickness of the damping layer. The results are respectively illustrated in Figs. 9c and 9d. As observed, the wider and thicker the damping layer, the higher the ENR. However, that comes to a limit. For instance, it is not recommended to attach very thick damping layers to the $\mathrm{ABH}$, because added stiffness may overcome the advantage of added mass (see how the ABH with $h_{v}=6 h_{r}$ does not always perform better than the one with $h_{v}=4 h_{r}$ in Fig. $9 \mathrm{~d}$ ). In fact, different strategies could be followed to find the right amount of damping. For example, in [58] critical coupling was exploited to find optimum damping distribution on an ABH beam. More standard optimization strategies were followed in [59] aiming at the same goal. On the other hand, optimization in the SmEdA framework has only started very recently (see e.g., [60]) but in the next future one would expect optimization techniques to be applied to problems as the one addressed in this work.

\section{Conclusions}

This paper has started investigating the performance of plates with embedded acoustic black holes $(\mathrm{ABHs})$ as part of more complex systems, in the mid-high frequency range. In particular, the energy noise reduction (ENR) between two cavities separated by an $\mathrm{ABH}$ plate has been analyzed using statistical modal 

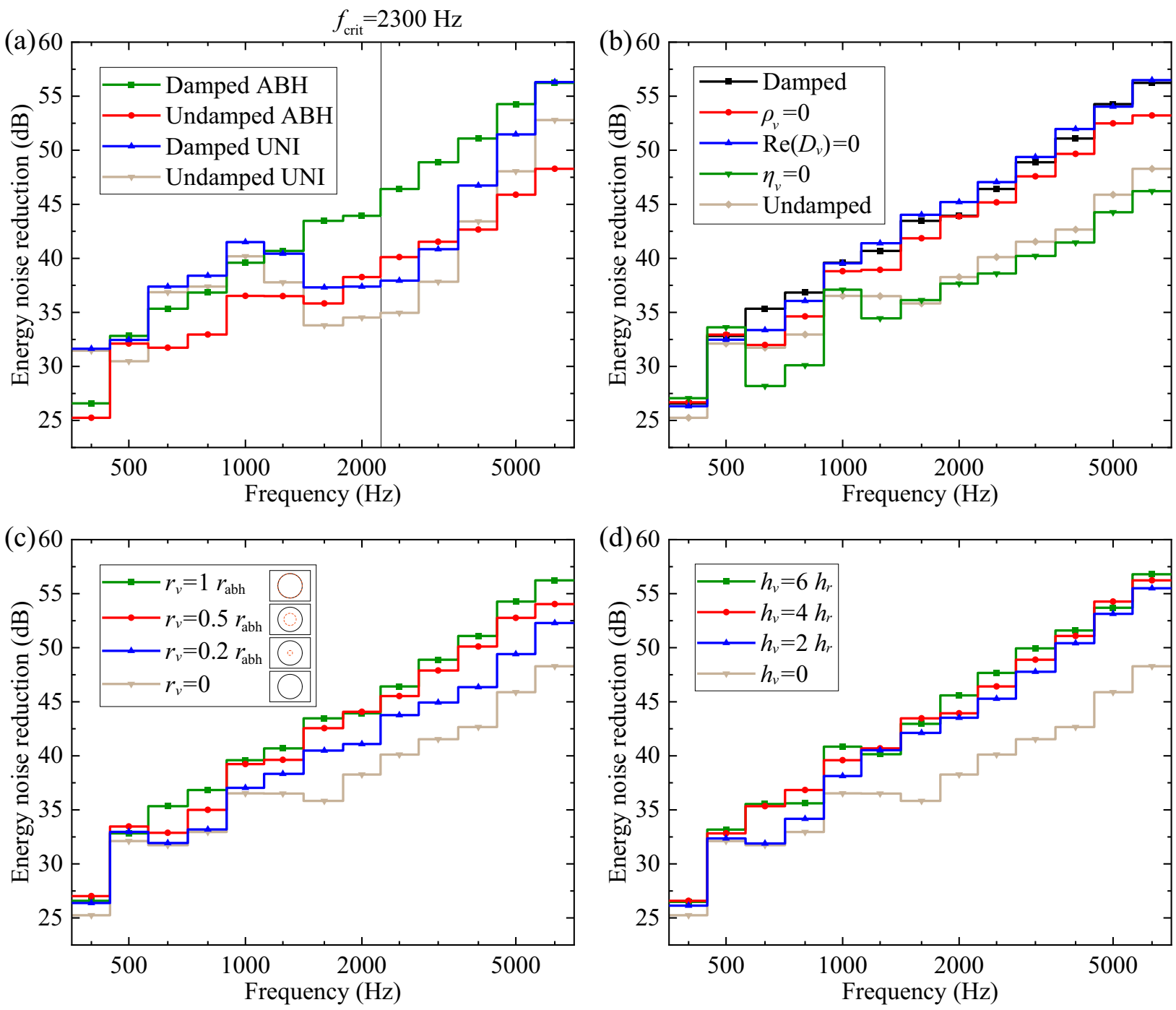

Figure 9: (a) The influence of damping layers on energy noise reduction (ENR). (b) Influence of added mass, added stiffness and energy dissipation by damping on ENR. (c) Effects of damping layer radius on ENR. (d) Effects of damping layer thickness on ENR.

energy distribution analysis (SmEdA). To compute the ABH plate modes needed in SmEdA use has been made of the Gaussian expansion method (GEM), while the modes of the cavities have been obtained from standard analytical formulas. Both resonant and non-resonant transmission between cavities have been considered. Simulations have shown that neglecting the latter may result in severe errors at low frequencies because of the strong modal interaction work at the separating $\mathrm{ABH}$ plate.

It has been demonstrated that ABH indentations can substantially increase the ENR of uniform plates. This occurs for two reasons: the reduction of plate vibrations due to the $\mathrm{ABH}$ effect and the lowering in radiation efficiency when waves propagating through the $\mathrm{ABH}$ become subsonic. The main advantage of $\mathrm{ABH}$ plates is that they avoid the ENR drop of uniform plates at the critical frequency, while the main disadvantage is mass decrease. The latter can worsen the ENR at lower frequencies. Therefore, a proper balance is always necessary when designing $\mathrm{ABH}$ plates for transmission loss purposes. A parametric analysis evaluating the influence of residual thickness, $\mathrm{ABH}$ radius and $\mathrm{ABH}$ order for a single $\mathrm{ABH}$ plate has confirmed this point. The values of the parameters must be carefully selected to compensate the problem 
of mass reduction with the benefits of the ABH effect. The same occurs when one considers embedding multiple ABHs on a plate. On the other hand, if for structural reasons it is decided to split a big ABH into smaller ones keeping the same amount of total $\mathrm{ABH}$ area and mass reduction, one should bear in mind that the smoothness cut-on frequency will increase and may become too large to prevent the critical frequency drop, if the ABHs get too small. Finally, we have examined the role played by the damping layer showing that its added mass slightly helps increasing the ENR while its added stiffness has the opposite effect. Its main contribution, however, is obviously that of energy dissipation without which the ABH effect becomes unnoticeable.

\section{Acknowledgements}

This work has been completed while the first author was performing a two-year PhD stay at La Salle, Universitat Ramon Llull, funded by the National Natural Science Foundation of China under Grant (51875061) and the China Scholarship Council (CSC No.201806050075). The authors gratefully acknowledge this support as well as the in-kind assistance from La Salle, Universitat Ramon Llull, and the Chongqing University to make that collaboration possible.

\section{Appendix A. SmEdA matrix including resonant and non-resonant transmission}

Matrix $\boldsymbol{\beta}$ in Eq. (31) is identified with that in Eq. (30), namely,

$$
\boldsymbol{\beta} \equiv\left[\begin{array}{ccc}
\boldsymbol{\beta}_{11} & \boldsymbol{\beta}_{12} & \boldsymbol{\beta}_{13} \\
\boldsymbol{\beta}_{12}^{\top} & \boldsymbol{\beta}_{22} & \boldsymbol{\beta}_{23} \\
\boldsymbol{\beta}_{13}^{\top} & \boldsymbol{\beta}_{23}^{\top} & \boldsymbol{\beta}_{33}
\end{array}\right]
$$

with diagonal entries,

$$
\begin{aligned}
& \boldsymbol{\beta}_{11}=\operatorname{diag}\left(\omega_{p} \eta_{p}+\sum_{q \in \hat{Q}^{\mathrm{R}}} \beta_{p q}+\sum_{r \in \hat{R}} \beta_{p r}\right)_{\hat{P} \times \hat{P}}, \\
& \boldsymbol{\beta}_{22}=\operatorname{diag}\left(\omega_{q} \eta_{q}+\sum_{p \in \hat{P}} \beta_{p q}+\sum_{r \in \hat{R}} \beta_{q r}\right)_{\hat{Q}^{\mathrm{R}} \times \hat{Q}^{\mathrm{R}}}, \\
& \boldsymbol{\beta}_{33}=\operatorname{diag}\left(\omega_{r} \eta_{r}+\sum_{p \in \hat{P}} \beta_{p r}+\sum_{r \in \hat{R}} \beta_{q r}\right)_{\hat{R} \times \hat{R}},
\end{aligned}
$$

and off-diagonal entries

$$
\boldsymbol{\beta}_{12}=-\left[\beta_{p q}\right]_{\hat{P} \times \hat{Q}^{\mathrm{R}}}, \quad \boldsymbol{\beta}_{13}=-\left[\beta_{p r}\right]_{\hat{P} \times \hat{R}} \text {, and } \boldsymbol{\beta}_{23}=-\left[\beta_{q r}\right]_{\hat{Q}^{\mathrm{R}} \times \hat{R}} .
$$

In the special case of non-resonant transmission being neglected, $\boldsymbol{\beta}_{13}=\mathbf{0}$ and the above expressions simplify to

$$
\boldsymbol{\beta}_{11}=\operatorname{diag}\left(\omega_{p} \eta_{p}+\sum_{q \in \hat{Q}^{\mathrm{R}}} \beta_{p q}\right)_{\hat{P} \times \hat{P}},
$$




$$
\boldsymbol{\beta}_{22}=\operatorname{diag}\left(\omega_{q} \eta_{q}+\sum_{p \in \hat{P}} \beta_{p q}+\sum_{r \in \hat{R}} \beta_{q r}\right)_{\hat{Q}^{\mathrm{R}} \times \hat{Q}^{\mathrm{R}}},
$$

and

$$
\boldsymbol{\beta}_{33}=\operatorname{diag}\left(\omega_{r} \eta_{r}+\sum_{r \in \hat{R}} \beta_{q r}\right)_{\hat{R} \times \hat{R}},
$$

for the diagonal terms, and to

$$
\boldsymbol{\beta}_{12}=-\left[\beta_{p q}\right]_{\hat{P} \times \hat{Q}^{\mathrm{R}}} \text {, and } \boldsymbol{\beta}_{23}=-\left[\beta_{q r}\right]_{\hat{Q}^{\mathrm{R}} \times \hat{R}},
$$

for the off-diagonal ones.

\section{References}

[1] M. Mironov, Propagation of a flexural wave in a plate whose thickness decreases smoothly to zero in a finite interval, Sov. Phys. Acoust. 34 (1988) 318-319.

[2] V. Krylov, F. Tilman, Acoustic black holes for flexural waves as effective vibration dampers, J. Sound Vib. 274 (2004) 605-619.

[3] V. Krylov, New type of vibration dampers utilising the effect of acoustic'black holes', Acta Acust. united Ac. 90 (2004) 830-837.

[4] M. Mironov, V. Pislyakov, One-dimensional acoustic waves in retarding structures with propagation velocity tending to zero, Acoust. Phys. 48 (2002) 347-352.

[5] O. Guasch, M. Arnela, P. Sánchez-Martín, Transfer matrices to characterize linear and quadratic acoustic black holes in duct terminations, J. Sound Vib. 395 (2017) 65-79.

[6] O. Guasch, P. Sánchez-Martín, D. Ghilardi, Application of the transfer matrix approximation for wave propagation in a metafluid representing an acoustic black hole duct termination, Appl. Math. Model. 77 (2020) 1881-1893.

[7] J. P. Hollkamp, F. Semperlotti, Application of fractional order operators to the simulation of ducts with acoustic black hole terminations, J. Sound Vib. 465 (2020) 115035.

[8] V. Denis, A. Pelat, F. Gautier, B. Elie, Modal overlap factor of a beam with an acoustic black hole termination, J. Sound Vib. 333 (2014) 2475-2488.

[9] V. Denis, F. Gautier, A. Pelat, J. Poittevin, Measurement and modelling of the reflection coefficient of an acoustic black hole termination, J. Sound Vib. 349 (2015) 67-79.

[10] J. Deng, L. Zheng, P. Zeng, Y. Zuo, O. Guasch, Passive constrained viscoelastic layers to improve the efficiency of truncated acoustic black holes in beams, Mech. Syst. Signal Pr. 118 (2019) 461-476.

[11] L. Tang, L. Cheng, H. Ji, J. Qiu, Characterization of acoustic black hole effect using a one-dimensional fully-coupled and wavelet-decomposed semi-analytical model, J. Sound Vib. 374 (2016) 172-184.

[12] W. Huang, H. Ji, J. Qiu, L. Cheng, Analysis of ray trajectories of flexural waves propagating over generalized acoustic black hole indentations, J. Sound Vib. 417 (2018) 216-226.

[13] L. Ma, S. Zhang, L. Cheng, A 2D Daubechies wavelet model on the vibration of rectangular plates containing strip indentations with a parabolic thickness profile, J. Sound Vib. 429 (2018) 130-146.

[14] H. Li, C. Touzé, A. Pelat, F. Gautier, X. Kong, A vibro-impact acoustic black hole for passive damping of flexural beam vibrations, J. Sound Vib. 450 (2019) 28-46.

[15] P. Zeng, L. Zheng, J. Deng, A. Elsabbagh, S. Xiang, T. Yan, Y. Wu, Flexural wave concentration in tapered cylindrical beams and wedge-like rectangular beams with power-law thickness, J. Sound Vib. 452 (2019) 82-96.

[16] L. Tang, L. Cheng, Periodic plates with tunneled Acoustic-Black-Holes for directional band gap generation, Mech. Syst. Signal Pr. 133 (2019) 106257.

[17] S. Conlon, J. Fahnline, F. Semperlotti, Numerical analysis of the vibroacoustic properties of plates with embedded grids of acoustic black holes, J. Acoust. Soc. Am. 137 (2015) 447-457.

[18] X. Li, Q. Ding, Sound radiation of a beam with a wedge-shaped edge embedding acoustic black hole feature, J. Sound Vib. 439 (2019) 287-299.

[19] L. Ma, L. Cheng, Sound radiation and transonic boundaries of a plate with an acoustic black hole, J. Acoust. Soc. Am. 145 (2019) 164-172.

[20] L. Tang, L. Cheng, Impaired sound radiation in plates with periodic tunneled Acoustic Black Holes, Mech. Syst. Signal Pr. 135 (2020) 106410

[21] H. Zhu, F. Semperlotti, Phononic thin plates with embedded acoustic black holes, Phys. Rev. B 91 (2015) 104304. 
[22] H. Zhu, F. Semperlotti, Two-dimensional structure-embedded acoustic lenses based on periodic acoustic black holes, J. Appl. Phys. 122 (2017) 065104.

[23] S. S. Ganti, T.-W. Liu, F. Semperlotti, Topological edge states in phononic plates with embedded acoustic black holes, J. Sound Vib. (2019) 115060.

[24] V. Denis, A. Pelat, F. Gautier, Scattering effects induced by imperfections on an acoustic black hole placed at a structural waveguide termination, J. Sound Vib. 362 (2016) 56-71.

[25] L. Ma, L. Cheng, Numerical and experimental benchmark solutions on vibration and sound radiation of an acoustic black hole plate, Appl. Acoust. 163 (2020) 107223.

[26] D. O'Boy, V. Krylov, Vibration of a rectangular plate with a central power-law profiled groove by the Rayleigh-Ritz method, Appl. Acoust. 104 (2016) 24-32.

[27] J. Deng, L. Zheng, O. Guasch, H. Wu, P. Zeng, Y. Zuo, Gaussian expansion for the vibration analysis of plates with multiple acoustic black holes indentations, Mech. Syst. Signal Pr. 131 (2019) 317-334.

[28] R. Lyon, R. DeJong, Theory and Application of Statistical Energy Analysis, RH Lyon Corp, Cambridge MA, 2nd Edition, 1998.

[29] R.J.M Craik, Sound Transmission Trough Buildings Using Statistical Energy Analysis, Gower, London, 1996.

[30] A. Le Bot, Foundation of statistical energy analysis in vibroacoustics, OUP Oxford, 2015.

[31] L. Maxit, J. L. Guyader, Estimation of SEA coupling loss factors using a dual formulation and FEM modal information, part I: theory, J. Sound Vib. 239 (2001) 907-930.

[32] L. Maxit, J. L. Guyader, Estimation of SEA coupling loss factors using a dual formulation and FEM modal information, part II: numerical applications, J. Sound Vib. 239 (2001) 931-948.

[33] H. Ji, X. Wang, J. Qiu, L. Cheng, Y. Wu, C. Zhang, Noise reduction inside a cavity coupled to a flexible plate with embedded 2-D acoustic black holes, J. Sound Vib. 455 (2019) 324-338.

[34] X. Wang, H. Ji, J. Qiu, L. Cheng, Wavenumber domain analyses of vibro-acoustic decoupling and noise attenuation in a plate-cavity system enclosed by an acoustic black hole plate, J. Acoust. Soc. Am. 146 (2019) $72-84$.

[35] P. A. Feurtado, S. C. Conlon, Transmission loss of plates with embedded acoustic black holes, J. Acoust. Soc. Am. 142 (2017) 1390-1398.

[36] X. Du, D. Huang, Q. Fu, J. Zhang, Effects of acoustic black hole parameters and damping layer on sound insulation performance of abh circular plate, Appl. Sci. 9 (2019) 5366.

[37] J. Guyader, C. Boisson, C. Lesueur, Energy transmission in finite coupled plates, part I: Theory, J. Sound Vib. 81 (1982) $81-92$.

[38] B. Mace, Statistical energy analysis, energy distribution models and system modes, J. Sound Vib. 264 (2003) $391-409$.

[39] S. De Rosa, F. Franco, A scaling procedure for the response of an isolated system with high modal overlap factor, Mech. Syst. Signal Pr. 22 (2008) 1549-1565.

[40] S. De Rosa, F. Franco, On the use of the asymptotic scaled modal analysis for time-harmonic structural analysis and for the prediction of coupling loss factors for similar systems, Mech. Syst. Signal Pr. 24 (2010) 455-480.

[41] P. J. Shorter, R. S. Langley, Vibro-acoustic analysis of complex systems, J. Sound Vib. 288 (2005) 669-699.

[42] G. Tanner, Dynamical energy analysis - determining wave energy distributions in vibro-acoustical structures in the highfrequency regime, J. Sound Vib. 320 (2009) 1023-1038.

[43] L. Maxit, J. L. Guyader, Extension of SEA model to subsystems with non-uniform modal energy distribution, J. Sound Vib. 265 (2003) 337-358.

[44] L. Maxit, O. Guasch, Energy-based reformulated Craig-Bampton method for multiple flexural subsystems connected at a junction with low impedance mismatch, Mech. Syst. Signal Pr. 119 (2019) 471-485.

[45] H. D. Hwang, L. Maxit, K. Ege, Y. Gerges, J.-L. Guyader, SmEdA vibro-acoustic modelling in the mid-frequency range including the effect of dissipative treatments, J. Sound Vib. 393 (2017) 187-215.

[46] D. Karnopp, Coupled vibratory-system analysis, using the dual formulation, J. Acoust. Soc. Am. 40 (1966) $380-384$.

[47] L. Maxit, Analysis of the modal energy distribution of an excited vibrating panel coupled with a heavy fluid cavity by a dual modal formulation, J. Sound Vib. 332 (2013) 6703-6724.

[48] L. Maxit, O. Guasch, A dual modal formulation for multiple flexural subsystems connected at a junction in energy-based models, Mech. Syst. Signal Pr. 119 (2019) 457-470.

[49] J. Deng, O. Guasch, L. Zheng, Ring-shaped acoustic black holes for broadband vibration isolation in plates, J. Sound Vib. 458 (2019) 109-122.

[50] L. Maxit, K. Ege, N. Totaro, J.-L. Guyader, Non resonant transmission modelling with statistical modal energy distribution analysis, J. Sound Vib. 333 (2014) 499-519.

[51] À. Aragonès, L. Maxit, O. Guasch, A graph theory approach to identify resonant and non-resonant transmission paths in statistical modal energy distribution analysis, J. Sound Vib. 350 (2015) 91-110.

[52] L. Cheng, Vibroacoustic modeling of mechanically coupled structures: Artificial spring technique applied to light and heavy mediums, Shock Vib. 3 (1996) 193-200.

[53] C. D. Johnson, D. A. Kienholz, Finite element prediction of damping in structures with constrained viscoelastic layers, AIAA J. 20 (1982) 1284-1290.

[54] B.-A. Ma, J.-F. He, A finite element analysis of viscoelastically damped sandwich plates, J. Sound Vib. 152 (1992) 107-123.

[55] T. Yamaguchi, Y. Kurosawa, H. Enomoto, Damped vibration analysis using finite element method with approximated modal damping for automotive double walls with a porous material, J. Sound Vib. 325 (2009) 436-450.

[56] F. Cura, A. Mura, F. Scarpa, Modal strain energy based methods for the analysis of complex patterned free layer damped plates, J. Vib. Control 18 (2012) 1291-1302. 
[57] P. Morse, K. Ingard, Theoretical acoustics, Princeton University Press, 1968.

[58] J. Leng, V. Romero-García, A. Pelat, R. Picó, J.-P. Groby, F. Gautier, Interpretation of the acoustic black hole effect based on the concept of critical coupling, J. Sound Vib. 471 (2020) 115199.

[59] C. A. McCormick, M. R. Shepherd, Optimization of an acoustic black hole vibration absorber at the end of a cantilever beam, J. Acoust. Soc. Am. 145 (2019) EL593-EL597.

[60] Y. Yu, G. Zhao, S. Ren, Design optimization of mid-frequency vibro-acoustic systems using a statistical modal energy distribution analysis model, Struct. Multidiscipl. Optim. 59 (2019) 1455-1470. 